\title{
Graphene-based mid-infrared photodetectors using metamaterials and related concepts
}

\author{
Ming Ye ${ }^{1, a)}$, Jiajia Zha ${ }^{2}$, Chaoliang $\operatorname{Tan}^{2}$, and Kenneth B. Crozier ${ }^{1,3,4, a)}$ \\ ${ }^{1}$ Department of Electrical and Electronic Engineering, University of Melbourne, Victoria 3010, \\ Australia \\ ${ }^{2}$ Department of Electrical Engineering, City University of Hong Kong, 83 Tat Chee Avenue, \\ Kowloon, Hong Kong \\ ${ }^{3}$ School of Physics, University of Melbourne, Victoria 3010, Australia \\ ${ }^{4}$ Australian Research Council (ARC) Centre of Excellence for Transformative Meta-Optical \\ Systems, University of Melbourne, Victoria 3010, Australia \\ a) Authors to whom correspondence should be addressed: yeming3010@gmail.com; and \\ kcrozier@unimelb.edu.au
}

\begin{abstract}
Graphene, a semimetal with a gapless band structure, has been used in mid-infrared (MIR) photodetectors (PDs) for some time. However, these detectors often suffer from low responsivity due to the intrinsically low absorption and ultrashort carrier lifetime in graphene, large dark current and low detectivity due to the semi-metallic nature of graphene. Over the past decade, much effort has been devoted to addressing these issues. A variety of metamaterials and related concepts have been employed to improve the detector responsivity by enhancing the graphene absorption and/or the carrier collection efficiency. Here, we provide an overview of the graphene MIR PDs both with and without the use of approaches for responsivity enhancement. We focus our attention on the state-ofthe-art graphene MIR PDs whose performance is improved by employing metamaterials and related concepts, including band structure engineering, the photogating effect, integration with plasmonic nanostructures and waveguides, the use of asymmetric plasmons, coupled plasmon-phonon polaritons and small-twist-angle bilayer graphene. We conclude by providing possible directions for further
\end{abstract}


performance improvement of graphene MIR PDs and a discussion on future applications of these detectors.

\section{TABLE OF CONTENTS}

\section{INTRODUCTION \\ II. MID-INFRARED GRAPHENE PHOTODETECTORS WITHOUT RESPONSIVITY ENHANCEMENT}

A. Photothermoelectric effect

B. Photovoltaic effect

C. Pyroresistive effect

III. IMPROVING THE RESPONSIVITY OF MID-INFRARED GRAPHENE PHOTODETECTORS USING METAMATERIALS AND RELATED CONCEPTS

A. Band structure engineering of graphene

B. Introduction of photogating effect

C. Integration with plasmonic nanoantennas/nanoparticles/engineered electrodes

D. Integration with silicon waveguide

E. Enhanced PTE effect with asymmetric plasmons

F. Enhanced PTE effect in graphene p-n junction by coupling plasmonic antenna to hyperbolic phonon-polaritons (HPPs) in hexagonal boron nitride (h-BN)

G. Small-twist-angle bilayer graphene

IV. OUTLOOK AND CONCLUSIONS

\section{INTRODUCTION}

Photodetectors (PDs) convert photon signals into electrical signals, and are key components of modern communication and sensing technologies that play an important role in our daily lives. The development of PDs operating in the visible and near-infrared spectral ranges has reached a high level of maturity due to advances in materials, large-scale production and integration with complementary metal-oxide-semiconductor (CMOS) systems. High-performance and low-cost PDs with CMOS integrability have been widely deployed in the visible and near-infrared (NIR) spectral range, using materials including silicon, germanium and compounds such as gallium arsenide and indium phosphide. ${ }^{1}$ In contrast, room-temperature PDs operating in the mid-infrared (MIR, wavelength: $2.5-$ $25 \mu \mathrm{m})^{2}$ spectral range are less advanced in terms of integration and pixel counts, despite the important roles that they could play in a wide range of applications, including biosensing, ${ }^{3}$ security, ${ }^{4}$ 
spectroscopy ${ }^{5}$, thermal imaging, ${ }^{6}$ gas sensing, ${ }^{7}$ motion detection ${ }^{8}$ and so on. Mature technologies for MIR detection based on epitaxially-grown narrow-bandgap semiconductors, such as mercury cadmium telluride $(\mathrm{HgCdTe}),{ }^{9}$ indium antimonide $(\mathrm{InSb}){ }^{10}$ and lead selenide $(\mathrm{PbSe}),{ }^{11}$ still face challenges, such as the complex and costly fabrication processes, the need of cryogenic cooling to achieve high performance and lack of CMOS compatibility. In addition, these narrow-bandgap semiconductors are toxic and hazardous to the environment. Therefore, it remains a challenge to develop high-performance, low-cost, CMOS integrable and environment-friendly PDs operating in the MIR spectral region.

Recently, much effort has been invested in the search for new materials to address the aforementioned issues related to MIR PDs. This review will focus on the promising material platform of atomically thin graphene. Graphene provides opportunities for MIR PDs with broadband response and high-speed operation due to its unique electronic and optical properties. ${ }^{12}$ For example, the gapless electronic structure of graphene renders broadband photoresponse, with PDs reported spanning from the visible to the terahertz. ${ }^{13,14}$ High-speed PDs with GHz bandwidths have also been demonstrated, enabled by the ultrashort carrier lifetime of graphene. ${ }^{14,15}$ At MIR wavelengths, it is well established that reducing the volume of the active material while maintaining the absorbed optical power facilitates increased detectivity, a method known as "optical immersion". ${ }^{16}$ Graphene in some sense takes the concept of volume reduction to the limit as it is atomically thin. In addition to the advantages mentioned above, graphene also possesses several unique properties that are unmatched by most conventional semiconductor materials. For example, graphene is flexible, transparent and compatible with CMOS technologies, and thus holds great promise for developing wearable optoelectronic devices that can be integrated with CMOS read-out circuits. ${ }^{17}$ Due to its linear electronic dispersion, ${ }^{18}$ the Fermi level of graphene and the induced optical response can be strongly modulated via electrostatic gating, ${ }^{19}$ which provides an opportunity to achieve gate-tunable photoresponse, e.g. via electrical tuning of the graphene absorption. Despite these appealing 
properties, one of the drawbacks of graphene PDs is the large dark current that traverses the graphene channel when an external bias is applied, due to the semi-metallic nature of graphene. This imposes a limit on the achievable detectivity. However, it could be potentially addressed by forming heterostructures between graphene and other materials, thanks to the self-terminating lattices of graphene. Finally, graphene films can be readily grown by chemical vapor deposition (CVD) methods at low cost and are environmentally friendly, which addresses the aforementioned environmental issues of conventional narrow-bandgap semiconductors. These advantages of graphene make it a promising material for construction of MIR PDs. However, many of the early demonstrations of graphene MIR PDs suffered from low responsivity, ${ }^{20,21}$ due to the low optical absorption $(<2.3 \%)$ and short photocarrier lifetime (sub-picosecond) of this material. To improve the responsivity of graphene MIR PDs, various strategies have been developed including the use of metamaterials and related concepts to increase the optical absorption and/or carrier collection efficiency in graphene. A comparison of the performance of representative conventional MIR PDs and graphene MIR PDs is presented in Table I below. It can be seen that commercially available MIR PDs ${ }^{22-24}$ have high responsivities (of thousands of $\mathrm{V} / \mathrm{W}^{22,24}$ or several $\mathrm{A} / \mathrm{W}^{23}$ ) and detectivities of $10^{10}-10^{11} \mathrm{Jones}$. However, cryogenic cooling is often needed to achieve this high performance. ${ }^{22,23}$ Some recently reported graphene MIR PDs employing responsivity enhancement approaches (listed in Table I below) have achieved responsivities that are on par or even higher than those of the commercial MIR PDs. The graphene MIR PDs also show high speed and broad spectral response, for example Ref [49] reported a graphene PD with engineered electrodes with a response time of less than 20 pico-seconds and a spectral range from $0.8-20 \mu \mathrm{m}$. It is important to note that the performance metrics reported for the graphene MIR PDs in Table 1 were obtained at room temperature. Although graphene MIR PDs generally suffer from low detectivity due to large dark current, the devices included in Table 1 show detectivities that are comparable to that of the conventional MIR PDs operating at room temperature, e.g., both show detectivities on the order of $10^{9}$ Jones. 
TABLE I. Performance comparison of conventional and graphene-based MIR PDs

\begin{tabular}{|c|c|c|c|c|c|c|}
\hline \multirow[t]{2}{*}{ PD type } & \multicolumn{5}{|c|}{ Figures-of-merit } & \multirow[t]{2}{*}{ Ref. } \\
\hline & Responsivity & $\begin{array}{l}\text { Response } \\
\text { time }\end{array}$ & $\begin{array}{c}\text { Detectivity } \\
\text { (Jones) }\end{array}$ & $\begin{array}{l}\text { Spectral } \\
\text { range }\end{array}$ & $\begin{array}{c}\text { Operating } \\
\text { temperature }\end{array}$ & \\
\hline \multicolumn{7}{|l|}{ Conventional MIR PDs } \\
\hline $\begin{array}{c}\mathrm{HgCdTe} \\
\text { (InfraRed Associates) }\end{array}$ & $\sim 2000$ V/W & $\sim 0.4 \mu \mathrm{s}$ & $\sim 4 \mathrm{E} 10$ & $2-13 \mu \mathrm{m}$ & $77 \mathrm{~K}$ & 22 \\
\hline $\begin{array}{c}\text { InSb } \\
\text { (InfraRed Associates) }\end{array}$ & $\sim 3 \mathrm{~A} / \mathrm{W}$ & / & $\sim 1 \mathrm{E} 11$ & $1-6 \mu \mathrm{m}$ & $77 \mathrm{~K}$ & 23 \\
\hline $\begin{array}{l}\text { PbSe } \\
\text { (Thorlabs) }\end{array}$ & $\sim 3000 \mathrm{~V} / \mathrm{W}$ & $\sim 10 \mu \mathrm{s}$ & $2.5 \mathrm{E} 9$ & $1.5-4.8 \mu \mathrm{m}$ & $298 \mathrm{~K}$ & 24 \\
\hline \multicolumn{7}{|l|}{$\begin{array}{c}\text { Graphene-based MIR } \\
\text { PDs }\end{array}$} \\
\hline $\begin{array}{l}\text { Graphene PD with } \\
\text { engineered electrodes }\end{array}$ & $2-11.5 \mathrm{~A} / \mathrm{W}$ & $<20 \mathrm{ps}$ & $1.5-30 \mathrm{E} 8$ & $0.8-20 \mu \mathrm{m}$ & RT & 49 \\
\hline Hybrid graphene $/ \mathrm{Ti}_{2} \mathrm{O}_{3}$ & $>100 \mathrm{~A} / \mathrm{W}$ & $1-3 \mathrm{~ms}$ & $2-7 \mathrm{E} 8$ & $4.5-10 \mu \mathrm{m}$ & $\mathrm{RT}$ & 44 \\
\hline $\begin{array}{l}\text { Graphene PD with } \\
\text { asymmetric hot carrier } \\
\text { generatio3 }\end{array}$ & $2900 \mathrm{~V} / \mathrm{W}$ & $\sim 100 \mathrm{~ns}$ & $1.1 \mathrm{E} 9$ & $8-12 \mu \mathrm{m}$ & RT & 53 \\
\hline
\end{tabular}

In this Review, we focus on graphene-based PDs operating in the MIR wavelength range. We first discuss the graphene MIR PDs that do not use metamaterials or related concepts for responsivity enhancement. These PDs are categorized by detection mechanisms that include the photothermoelectric effect, photovoltaic effect and pyroresistive effect. We then review the graphene MIR PDs that employ metamaterials and related methods for improved responsivity. Finally, we conclude with our thoughts on fruitful future research areas, including further performance enhancements and new applications.

\section{MID-INFRARED GRAPHENE PHOTODETECTORS WITHOUT RESPONSIVITY ENHANCEMENT}

Despite the challenges of low optical absorption, ultra-short carrier lifetime and large dark current discussed in the previous section, graphene-based PDs have been successfully demonstrated in the MIR region. In this section, we review some of the key reports, categorized by detection mechanisms, including the photothermoelectric (PTE) effect, photovoltaic effect and pyroresistive 
effect. We note that bolometric effect in graphene has been employed to achieve MIR photodetection. However, the use of such bolometers in applications is restricted by their somewhat slow response and these devices are therefore not discussed in this review.

\section{A. Photothermoelectric effect}

Hot-carrier-assisted transport can play a dominant role in graphene-based photodetection, due to the carrier multiplication and the high carrier mobility of graphene..$^{20,25}$ It has been noted that, due to strong electron-electron interactions,${ }^{26}$ the photoexcited electron-hole pairs produce hot carriers in graphene within an ultra-short timescale of $\sim 10-50$ fs. ${ }^{27}$ The hot carriers remain at a temperature $\left(T_{e}\right)$ higher than the lattice for several picoseconds and slowly reach an equilibrium with the lattice on a nanosecond scale via scattering between the charge carriers and acoustic phonons. ${ }^{28}$ In graphene, the photothermoelectric (PTE) effect enables a Seebeck voltage arising from the light-induced temperature gradient via hot-carrier excitation. Graphene PDs based on the PTE effect have been demonstrated in the MIR region, with temperature gradient being generated by direct light absorption in the graphene, ${ }^{29}$ by light-excited graphene plasmons ${ }^{30,31}$ or by light-excited substrate phonons ${ }^{21}$.

Figures 1(a)-1(c) show graphene PDs based on the PTE effect in graphene $\mathrm{p}-\mathrm{n}$ junctions. ${ }^{29}$ The device shown in the top panel of Fig. 1(a) consists of a graphene channel that is half-covered by a poly(methyl methacrylate) (PMMA) mask. The graphene $\mathrm{p}-\mathrm{n}$ junction is formed by applying independent voltages on a nanopatterned electrolyte top gate and a $\mathrm{SiO}_{2}$ back gate. Due to the different Seebeck coefficients of the $\mathrm{p}$ - and $\mathrm{n}$-doped graphene regions, the light (wavelength: 1.55 $\mu \mathrm{m})$ absorption induced temperature gradient produces a net PTE photovoltage at the $\mathrm{p}-\mathrm{n}$ junction under zero bias at room temperature (Fig. 1(a), bottom panel). To optimize the photovoltage collection for incident illumination with a Gaussian profile, several $\mathrm{p}-\mathrm{n}$ junctions are fabricated and connected in series, forming a circular shape (left panel of Fig. 1(b)). The graphene segment is p- or $\mathrm{n}$-doped in an alternating fashion so that the generated photovoltages of neighboring $\mathrm{p}-\mathrm{n}$ junctions 
have opposite signs and can be added up to give the total photovoltage (equivalent circuit model in the right panel of Fig. 1(b)). A maximum room temperature responsivity of $26.2 \mathrm{mV} / \mathrm{W}$ is measured at $\lambda=8.58 \mu \mathrm{m}$ under zero bias, when eight voltage segments are included in the measurement, which is shown in Fig. 1(c).

Figures 1(d)-1(i) show a graphene photoconductor based on the plasmon-assisted PTE effect. $^{30}$ The graphene photoconductor (Fig. 1d) consists of graphene-disk plasmonic resonators (GDPRs) connected by quasi-1D graphene nanoribbons (GNRs). The GDPRs serve as the source of hot carriers as they support plasmonic resonance upon light illumination. Figs. 1(e)-1(h) illustrate two carrier transport mechanisms whose occurrence depends on whether resonant plasmons are excited. Without plasmon excitation, carriers transport is by nearest-neighbor hopping (NNH, Fig. 1(h)) between neighboring localized states, leading to a small thermal smearing of the carrier distribution (Fig. 1(e)). The excitation of plasmonic resonance produces hot carriers at elevated temperature (thermal carrier excitation (TCE), Fig. 1(g)), resulting in greater thermal smearing of the carrier distributions, which facilitates their transport through the potential barriers (Fig. 1(f)). Under the TCE regime, a room-temperature responsivity of $16 \mathrm{~mA} / \mathrm{W}$ (bias voltage $\mathrm{V}_{\mathrm{b}}=1 \mathrm{~V}$ ) is obtained at a wavelength of $12.2 \mu \mathrm{m}$ (Fig. 1(i)).

Figures 1(j)-1(1) show a graphene photoconductor based on the phonon-assisted PTE effect. ${ }^{21}$ A photoresponsivity of around $58.5 \mathrm{nA} / \mathrm{W}$ (under zero source-drain bias) is measured close to the edges of the electrodes at a frequency of $1080 \mathrm{~cm}^{-1}$ (wavelength of $9.26 \mu \mathrm{m}$ ) for incident light polarized parallel to the edge of the contact, as shown in Figs. 1(j) and 1(k). The photodetection is based on a phonon-assisted PTE effect, where the incident light is absorbed by the substrate phonons (transverse (TO) phonon band of $\mathrm{SiO}_{2}$ is centered around $1065.5 \mathrm{~cm}^{-1}$ ), leading to local heating of the charge carriers in graphene which generates a PTE photovoltage and causes a current flow. The phonon-assisted PTE effect can be verified by the fact that the device shows photocurrent across a large area, even when the light spot is outside the graphene region (Fig. 1(k)). When the excitation 
frequency is $1390 \mathrm{~cm}^{-1}$, i.e. far from the substrate TO phonon band, the device shows photocurrent in a much smaller area close to the edges of the contacts (Fig. 1(1)), since heating of the charge carriers in graphene is caused by direct light absorption in graphene and is limited by the laser spot size.
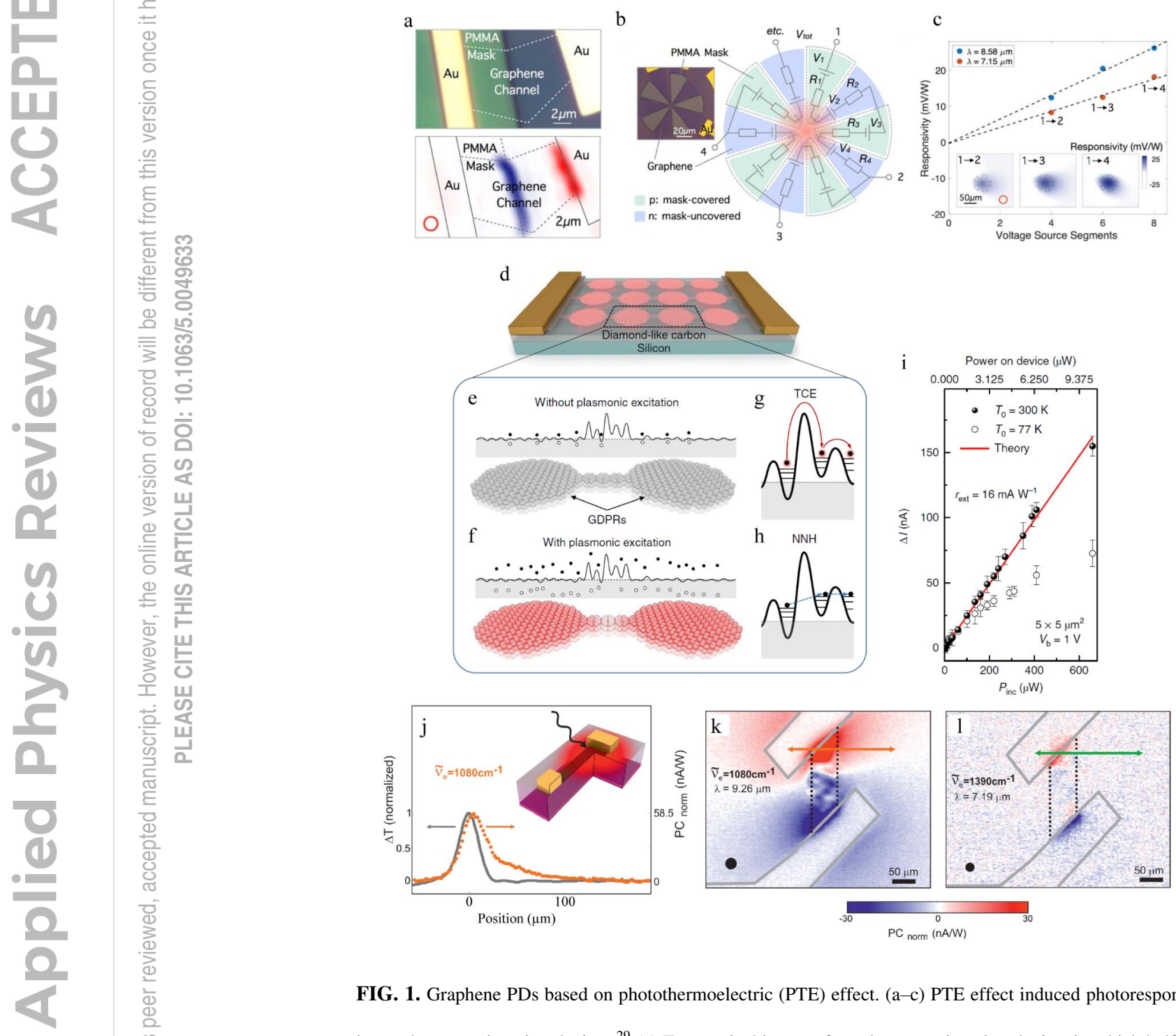

FIG. 1. Graphene PDs based on photothermoelectric (PTE) effect. (a-c) PTE effect induced photoresponse in graphene $\mathrm{p}-\mathrm{n}$ junction devices. ${ }^{29}$ (a) Top: optical image of graphene $\mathrm{p}-\mathrm{n}$ junction device, in which half of 
graphene channel is covered by PMMA mask. Bottom: photovoltage map of $\mathrm{p}-\mathrm{n}$ junction device measured at a NIR wavelength of $1.55 \mu \mathrm{m}$. Red circle indicates full width half maximum (FWHM) of laser spot $(\sim 1.7 \mu \mathrm{m})$. (b) Optical image (left) and equivalent circuit diagram (right) of graphene thermopile comprising multiple graphene $\mathrm{p}-\mathrm{n}$ junctions. Graphene is etched into a circular channel with a meandering shape, with each segment being p- or n-doped in an alternating fashion. (c) Responsivity of thermopile as a function of number of voltage source segments included in circuit at $\lambda=8.58 \mu \mathrm{m}$ and $\lambda=7.15 \mu \mathrm{m}$. Device is illuminated at its center by a Gaussian beam. Inset shows responsivity maps measured at $\lambda=8.58 \mu \mathrm{m}$, with 4,6 and 8 voltage source segments included in circuit, respectively. Red circle indicates FWHM of laser spot $(\sim 30 \mu \mathrm{m})$. Reproduced with permission from C. Peng et al., New J. Phys. 20, 083050 (2018). (d-i) Plasmon-assisted PTE effect in graphene photoconductor. ${ }^{30}$ (d) Schematic of device, which consists of graphene-disk plasmonic resonators (GDPRs, red circles) connected by quasi-1D graphene nanoribbons (GNRs). (e-f) Conceptual illustration of disorder potential (solid curve) around chemical potential and carrier thermal smearing before (e) and after (f) photoexcitation at room temperature. $(\mathrm{g}-\mathrm{h})$ Conceptual illustration of thermal-carrier excitation (TCE) transport (g), in which electrons with higher thermal energy can overcome localized potential barriers and nearest-neighbor hopping $(\mathrm{NNH})$ transport $(\mathrm{h})$, in which thermalized electrons evanescently hop between neighboring localized states under external driving electric field. (i) Photocurrent vs incident power for device with an area of $5 \times 5 \mu \mathrm{m}^{2}$. Top $\mathrm{x}$ axis shows power impinging on device area. Filled (open) symbols represent data acquired at $\mathrm{T}_{0}=300 \mathrm{~K}(77 \mathrm{~K})$. Red curve is theoretical result. Error bars represent standard deviation. Reproduced with permission from Q. S. Guo et al., Nat. Mater. 17, 986 (2018). Copyright 2018 Nature Publishing Group. (j-1) Phonon-assisted PTE effect in a graphene photoconductor on a polar substrate. ${ }^{21}$ (j) Photocurrent (orange curve) at $1080 \mathrm{~cm}^{-1}$ along orange arrow in (k). Gray curve represents laser spot size, as obtained from spatial derivative of optical transmission measurement. (k-1) Photocurrent maps upon excitation with wavelength $\lambda=9.26 \mu \mathrm{m}\left(1080 \mathrm{~cm}^{-1}\right)(\mathrm{k})$, and $\lambda=7.19 \mu \mathrm{m}\left(1390 \mathrm{~cm}^{-1}\right)(\mathrm{l})$. Gray lines depict contacts. Black dotted lines indicate graphene position. Black circle at bottom left represents beam spot size (FWHM $22 \mu \mathrm{m}(\mathrm{k})$ and $14 \mu \mathrm{m}(\mathrm{l}))$. Light polarization is parallel to contacts. Adapted with permission from M. Badioli et al., Nano Lett. 14, 6374 (2014). Copyright 2014 American Chemical Society. 


\section{B. Photovoltaic effect}

The photovoltaic effect involves the photoexcitation of charge carriers and their subsequent separation by built-in electric fields at junctions between graphene regions with electron doping and hole doping, or between graphene sections of same type of doping but with different doping levels. ${ }^{32,33}$ The built-in electric fields can be introduced by local chemical doping, ${ }^{34}$ by local electrostatic gating (via split gates) ${ }^{32,35}$ or by the metallic doping effect that arises from the workfunction difference between the contacting metal and graphene. ${ }^{32,33,36}$ Graphene PDs based on the photovoltaic effect can operate at zero source-drain bias voltage, though they often have low responsivities because charge separation is inefficient in the unbiased operation mode. A source-drain bias voltage can be applied to improve the charge separation by producing a much larger external electric field. However, this will also lead to a larger dark current which undermines the detector specific detectivity. It should be noted that due to the electron-electron scattering, ${ }^{37}$ there can be multiple electron-hole pairs excited for each absorbed photon (also known as carrier multiplication), ${ }^{38}$ leading to enhanced photodetection efficiency. Using the photovoltaic effect, room-temperature MIR photodetection has been demonstrated with a simple graphene field-effect transistor on $\mathrm{S}_{2} \mathrm{Si}_{2}(30$ $\mathrm{nm}$ thick)/Si/Au substrate (Fig. 2(a), top panel). ${ }^{39}$ As a result of the doping of graphene by the palladium contacts, built-in electric fields are formed near the contact regions (Fig. 2(a), bottom panel). Photocarriers excited near the contact regions will be separated and flow in opposite directions at the two electrodes depending on the direction of the electric field. Consequently, the photovoltages measured at the two electrodes have opposite signs, as shown in Fig. 2(b). The photovoltage measured when the laser illumination spot is in the center of the graphene channel is almost zero due to the short carrier lifetime and long transit time. 
a

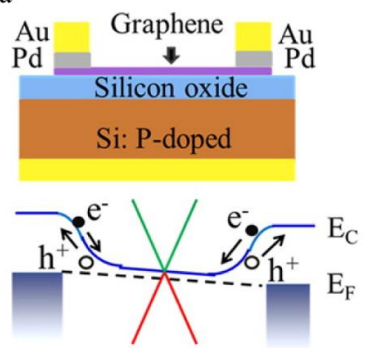

b

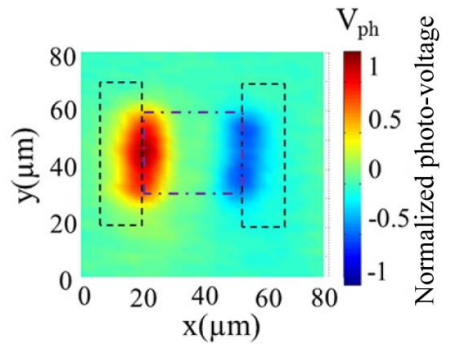

Fig. 2. Graphene PD based on photovoltaic effect. ${ }^{39}$ (a) Top: cross-section schematic of graphene field effect transistor on $\mathrm{SiO}_{2}(30 \mathrm{~nm}$ thick)/Si/Au substrate. Bottom: energy band diagram. Blue line indicates potential profile of conduction band edge $\left(\mathrm{E}_{\mathrm{C}}\right)$. (b) Photovoltage map of graphene PD of panel (a), measured with a continuous wave quantum cascade laser (wavelength: $4.45 \mu \mathrm{m}$ ). Source-drain bias voltage is $0.5 \mathrm{~V}$. Dashed lines and dashed-dotted lines indicate positions of contact pads and graphene sheet, respectively. Adapted with permission from Y. Yao et al., Nano Lett 14, 3749 (2014). Copyright 2014 American Chemical Society.

\section{Pyroresistive effect}

When a pyroelectric material is illuminated, heating resulting from light absorption and the resulted temperature increase will produce a variation of spontaneous polarization in the material due to the pyroelectric effect. In bringing a graphene sheet in direct contact with a pyroelectric material, the light-produced bound (polarization) surface charges in the pyroelectric material will induce free charges in the graphene, resulting in a change of the resistance of the graphene. This phenomenon has been termed as the pyroresistive effect and can be used in uncooled graphene MIR PDs. ${ }^{40,41}$ Figure 3 shows such an example in which room-temperature MIR photodetection is demonstrated by combining a dual-gated single-layer graphene (SLG) amplifier with a pyroelectric material. ${ }^{40}$ The device consists of a graphene field-effect transistor on a pyroelectric substrate (500 $\mu \mathrm{m}$-thick z-cut lithium niobate crystal, $\mathrm{LiNbO}_{3}$ ), as shown in Fig. 3(a). A 10 nm-thick $\mathrm{Al}_{2} \mathrm{O}_{3}$ dielectric layer isolates the graphene from an $\mathrm{H}$-shaped floating Au structure. The lateral pads of the floating $\mathrm{Au}$ structure 
are placed in direct contact with the substrate. The floating metallic structure is designed to concentrate the pyroelectric charges on the top-gate capacitor of the graphene channel, leading to a temperature coefficient of the resistance of up to $900 \% \mathrm{~K}^{-1}$. Figure 3(b) depicts the corresponding electrical model of the device. The gain of the SLG amplifier is controlled by the geometric ratio of the areas of the capacitors $\mathrm{C}_{3}$ and $\mathrm{C}_{2}$, which is a result of the conservation of charge. Consequently, the pyroelectric response of the $\mathrm{LiNbO}_{3}$ crystal is transduced with high gain (up to 200) into resistivity modulation for graphene. The modulation of the channel drain current under MIR radiation at 1100 $\mathrm{cm}^{-1}(\sim 9 \mu \mathrm{m})$ is shown in Fig. 3(c), revealing a responsivity of $0.27 \mathrm{~mA} / \mathrm{W}$ under a source-drain bias voltage of $10 \mathrm{mV}$. Figure 3(d) shows the photocurrent maps of the device measured by chopping the beam at different frequencies. The two peaks in the photocurrent map become progressively resolved as the frequency increases and match the location of the lateral pads when the frequency is above 500 Hz. This can be understood by the fact that at higher frequencies, there is less time for the heat to laterally spread from the illuminated spot to the pads, which translates into negligible charge doping of the graphene channel.

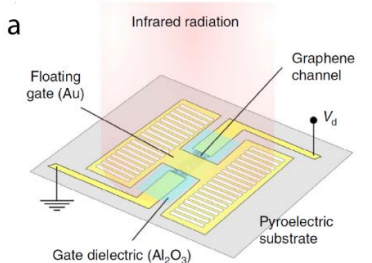

a Gate dielectric $\left(\mathrm{Al}_{2} \mathrm{O}_{3}\right)$

d

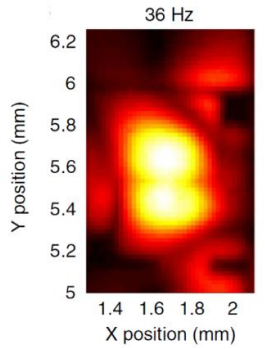

b

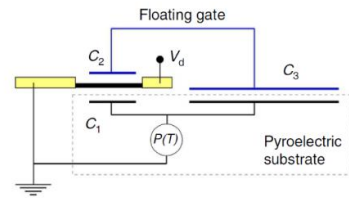

$128 \mathrm{~Hz}$
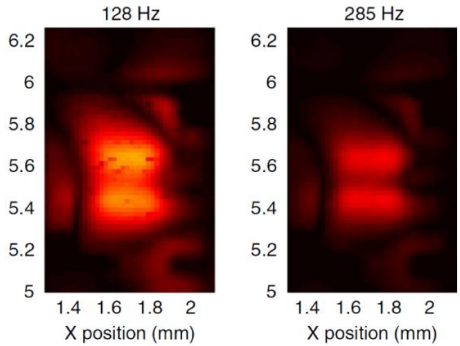
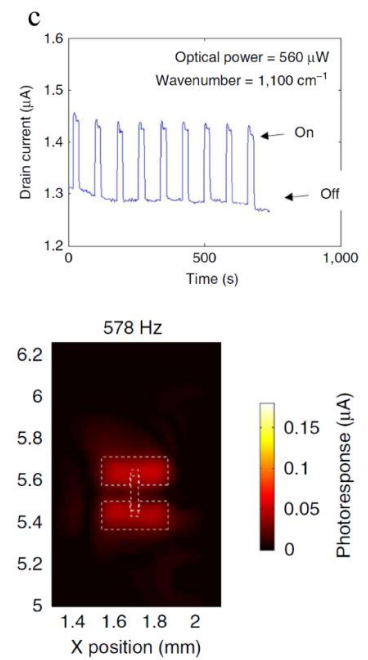
FIG. 3. Graphene PD based on pyroresistive effect. ${ }^{40}$ (a) Schematic of graphene PD consisting of a dual-gated SLG amplifier on a pyroelectric substrate (500 mm-thick z-cut lithium niobate crystal, $\mathrm{LiNbO}_{3}$ ). Floating metallic structure is fabricated on top of $\mathrm{Al}_{2} \mathrm{O}_{3}$ coated graphene channel and substrate to concentrate pyroelectric charges on top-gated graphene channel. (b) Electrical model of device of panel (a). (c) Modulated drain current under MIR illumination at $1100 \mathrm{~cm}^{-1}(\sim 9 \mu \mathrm{m})$ at source-drain bias voltage of $10 \mathrm{mV}$. (d) Photocurrent maps measured with illumination from MIR (frequency: $1100 \mathrm{~cm}^{-1}$ ) beam that is chopped at different frequencies. Reproduced with permission from U. Sassi et al., Nat. Commun. 8, 1 (2017). Licensed under a Creative Commons Attribution (CC-BY_4.0) license.

\section{IMPROVING THE RESPONSIVITY OF INFRARED GRAPHENE PHOTODETECTORS USING METAMATERIALS AND RELATED CONCEPTS}

Although graphene PDs have been successfully demonstrated using the detection mechanisms discussed in the previous section, their responsivities are modest. ${ }^{20,21}$ This originates from the intrinsically low optical absorption and the short photocarrier lifetime (sub-picosecond) of graphene. Recently, considerable efforts have thus been devoted to improving the responsivity of graphene MIR PDs using metamaterials and related concepts to enhance the optical absorption efficiency and/or carrier collection efficiency in graphene. In this section, we will discuss such devices. The approaches that they use include band structure engineering, ${ }^{42,43}$ the photogating effect, ${ }^{44-46}$ integration with plasmonic nanoantennas/nanoparticles/engineered electrodes, ${ }^{39,47-51}$ integration with waveguides, ${ }^{52}$ enhancement of PTE effect with asymmetric plasmons, ${ }^{53}$ enhancement of PTE effect with coupled plasmon-hyperbolic phonon polaritons $s^{54,55}$ and the use of small-twist-angle bilayer graphene ${ }^{56}$.

\section{A. Band structure engineering of graphene}




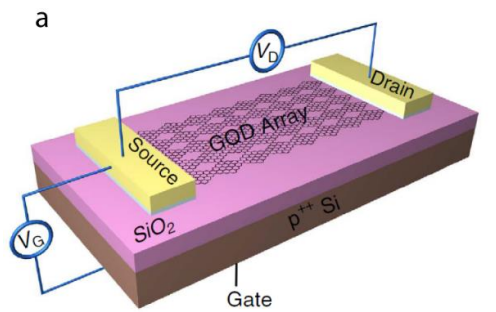

$\mathrm{b}$

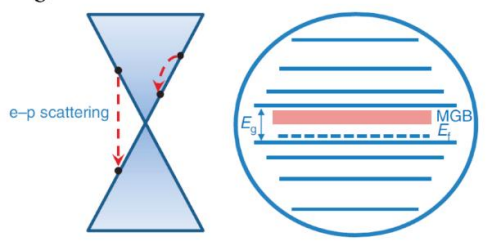

$\mathrm{c}$

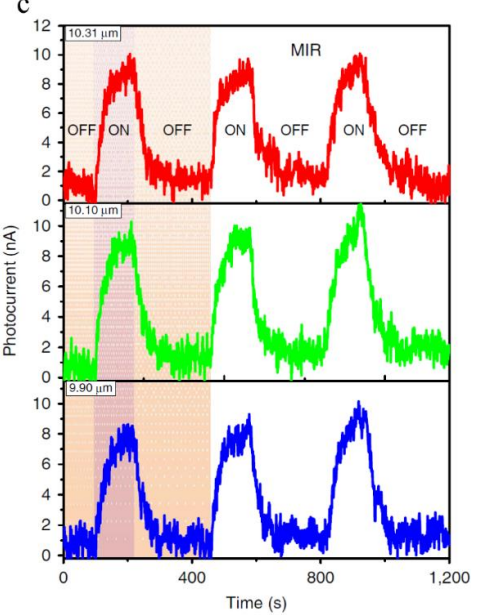

FIG. 4. Band structure engineering in a graphene quantum dot $\mathrm{PD}^{.2}$ (a) Schematic of graphene quantum dot PD. (b) Band structure diagram of pure graphene sheet (left) and graphene quantum dot array (right) with midgap-states band (red shaded region). Red dashed arrows indicate interband and intraband electron-phonon scattering. (c) Time-dependent photocurrent measured at MIR wavelengths close to $10 \mu \mathrm{m}$. Reproduced with permission from Y. Z. Zhang et al., Nat. Commun. 4, 1811 (2013).

Band structure engineering has been shown to enhance the responsivity of graphene-based PD by boosting the optical absorption by the graphene channel and by introducing photoconductive gain. For example, Zhang et al. demonstrate a graphene MIR PD that consists of a graphene quantum dot (GQD) array on a $\mathrm{SiO}_{2} / \mathrm{Si}$ substrate (Fig. 4). ${ }^{42}$ By patterning the graphene channel into GQD structures (Fig. 4 (a)), the band structure of graphene is significantly altered. As illustrated in the right panel of Fig. 4(b), a bandgap and a mid-gap-states band (MGB) are created due to quantum confinement effects. This is drastically different from the band structure of un-patterned SLG, which depicts the well-known Dirac cone shape (left panel of Fig. 4(b)). In un-patterned SLG, the lifetime of photogenerated electrons is usually very short due to the high interband and intraband electron- 
phonon scattering rates. In contrast, the photocarrier lifetime in the GQD structure is prolonged because electrons can be effectively trapped in the MGB which leads to the mitigation of electronphonon scattering. ${ }^{57}$ In addition, the existence of the MGB allows carrier multiplication to occur, thereby increasing the overall electron generation efficiency. The efficient electron generation and subsequent trapping in the MGB give rise to a very high photoconductive gain, which enables sensitive MIR photodetection with a measured responsivity of $0.4 \mathrm{~A} / \mathrm{W}$ under a source-drain bias voltage of $20 \mathrm{mV}$ (Fig. 4(c)). Unfortunately, the response time of the detector is very slow ( tens of seconds) due to the long lifetimes of the trapped electrons, as can be seen from Fig. 4(c). The detector operation is restricted to low temperature $(\sim 10 \mathrm{~K})$ because the responsivity reduces at higher temperatures due to shortened carrier lifetime.

\section{B. Introduction of photogating effect}

The photogating effect is a light-induced phenomenon generally involving carrier transfer of one type from a photosensitive material into a separate charge transport layer, while carriers of the other type accumulate in the photosensitive material and produce an electric field, leading to a conductance change of the transport layer. ${ }^{58}$ Photogating can significantly increase the internal quantum efficiency (IQE) of PDs through the increase in gain that comes from carriers being circulated around the electrical circuit multiple times before recombination. However, it should be noted that such an increase in IQE can come at the expense of slow response due to the long carrier lifetime. The photogating effect has been employed in graphene PDs to achieve high responsivities (> $1 \mathrm{AW}^{-1}$ ) at room temperature (Fig. 5) by either coupling graphene with narrow-bandgap semiconductor nanoparticles, ${ }^{44}$ narrow-bandgap topological insulators, ${ }^{45}$ or by introducing a tunneling barrier in a graphene double-layer heterostructure. ${ }^{46}$

By hybridizing graphene with a narrow-bandgap semiconductor, titanium sesquioxide $\left(\mathrm{Ti}_{2} \mathrm{O}_{3}\right.$, bandgap: $0.09 \mathrm{eV}$ ), room-temperature MIR PDs with high responsivity are reported, as shown in Figs. 
5(a)-5(c). ${ }^{44}$ Upon light absorption in $\mathrm{Ti}_{2} \mathrm{O}_{3}$, photo-generated electrons are trapped in the $\mathrm{Ti}_{2} \mathrm{O}_{3}$ nanoparticles while the holes are transferred into the graphene channel (Fig. 5(b)), resulting in a change of the graphene channel conductance that is then read out as photocurrent. High responsivity is achieved by the strong photoconductive gain due to the long lifetime ( several milliseconds) of the trapped electrons in the $\mathrm{Ti}_{2} \mathrm{O}_{3}$. Photocurrents are measured under laser illumination at a wavelength of $10 \mu \mathrm{m}$ for hybrid graphene/ $\mathrm{Ti}_{2} \mathrm{O}_{3}$ PDs with monolayer-, bilayer- and trilayer-graphene (Fig. 5(c)). A maximum room-temperature responsivity of $\sim 300 \mathrm{~A} / \mathrm{W}$ is obtained in a hybrid trilayer graphene $/ \mathrm{Ti}_{2} \mathrm{O}_{3}$ photodetector for illumination at $\lambda=10 \mu \mathrm{m}$ under source-drain voltage of $2 \mathrm{mV}$ (Fig. 5(c), blue curve). The detector response time is limited to around 1-3 ms however (Fig. 5(c)).

Based on a similar strategy, strong MIR photoresponse is reported in a graphene PD coupled with a narrow-bandgap topological insulator bismuth selenide $\left(\mathrm{Bi}_{2} \mathrm{Se}_{3}\right.$, bandgap: $\left.0.3 \mathrm{eV}\right)$, as shown in Figs. 5(d)-5(f). ${ }^{45}$ In this device, the $\mathrm{Bi}_{2} \mathrm{Se}_{3}$ layer serves as a broadband MIR absorber. Due to the asymmetric energy barrier at the $\mathrm{Bi}_{2} \mathrm{Se}_{3}$-graphene interface (Fig. 5(e)), the photoexcited holes are transferred to the graphene layer, while the photoexcited electrons remain in the $\mathrm{Bi}_{2} \mathrm{Se}_{3}$, leading to an electrostatic modulation of the carrier density in graphene, i.e. a photogating effect. Fig. 5(f) shows the measured transfer characteristics with and without MIR illumination $(\lambda=3.5 \mu \mathrm{m})$ under a sourcedrain bias voltage of $0.5 \mathrm{~V}$, revealing a strong photoresponse with a responsivity of $1.97 \mathrm{~A} / \mathrm{W}$. The large shift of the Dirac point under illumination indicates a strong modulation of the hole density in graphene, verifying the occurrence of the photogating effect.

Figures 5(g)-5(i) show an example of responsivity enhancement in a graphene double-layer heterostructure with a tunneling barrier using the photogating effect. ${ }^{46}$ The detector consists of two graphene layers that sandwich a thin tunneling barrier ( $\sim \mathrm{nm}$ thick intrinsic silicon layer), as shown in Fig. 5(g). Due to the fact that the two graphene layers have different doping levels, with the top graphene layer being more heavily p-doped than the bottom graphene layer, the energy band in the tunneling barrier is tilted toward the bottom graphene layer as a result of the equilibration of the Fermi 
level (Fig. 5(h)). Consequently, photoexcited electrons in the top graphene layer can tunnel through the barrier to the bottom layer, while photoexcited holes are accumulated in the top graphene layer, resulting in a strong photogating effect on the bottom graphene channel layer. As a result of the strong photogating effect, efficient MWIR photodetection at room temperature is demonstrated, with a maximum responsivity of around $1.1 \mathrm{~A} / \mathrm{W}$ under a source-drain bias voltage of $1.5 \mathrm{~V}$ at $\lambda=3.2 \mu \mathrm{m}$, rivaling that of the state-of-the-art MWIR detectors operating at room temperature. Again, the bandwidth of the detector is limited to a modest $10-1000 \mathrm{~Hz}$.

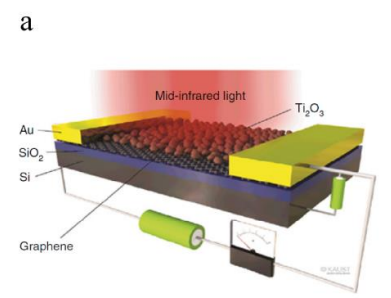

b

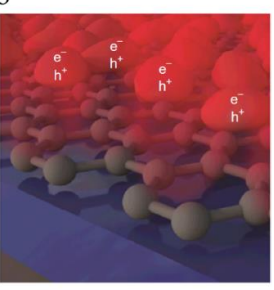

d

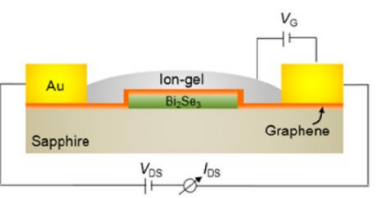

e
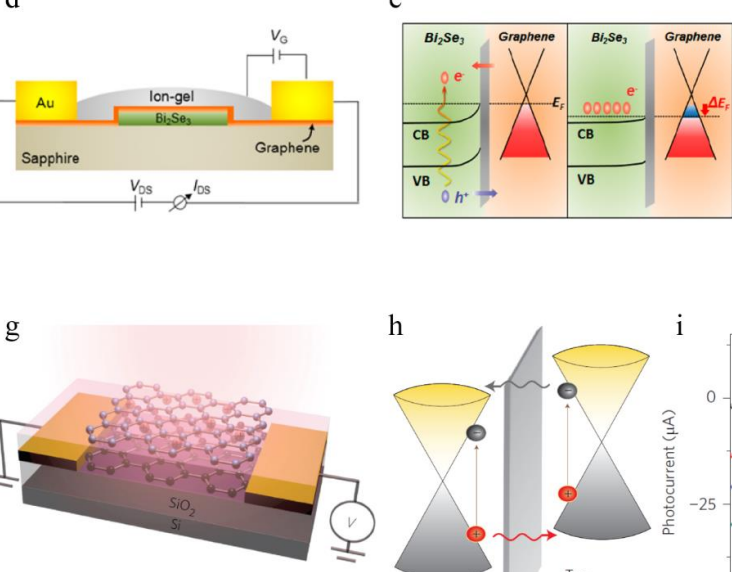

h

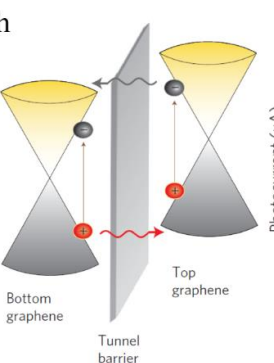

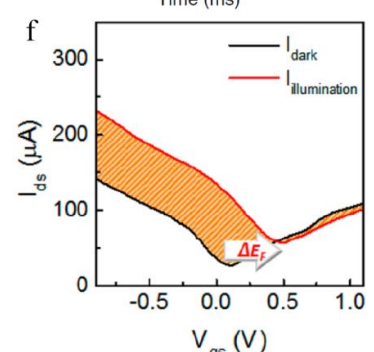

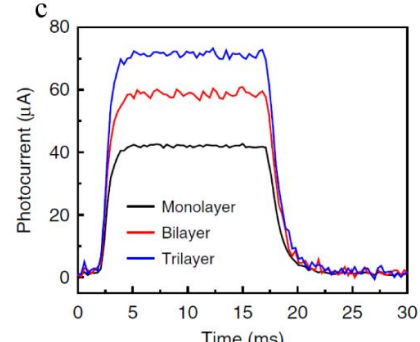

Time (ms)

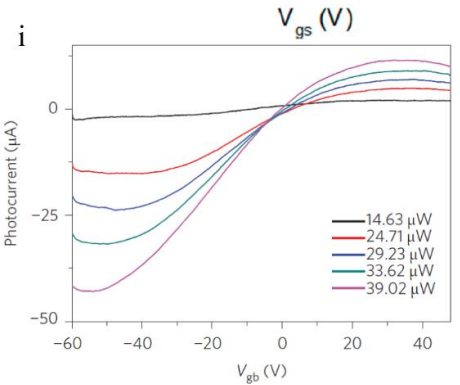

FIG. 5. Photogating effects for responsivity enhancement of MIR graphene PDs. (a-c) MIR photodetection by coupling graphene with narrow-bandgap semiconductor $\mathrm{Ti}_{2} \mathrm{O}_{3}{ }^{44}$ (a) Schematic of hybrid graphene/ $\mathrm{Ti}_{2} \mathrm{O}_{3}$ 
nanoparticle PD. (b) Cartoon illustration of charge transfer process occurring at graphene $/ \mathrm{Ti}_{2} \mathrm{O}_{3}$ interface. (c) Time-dependent photocurrent for photodetector illustrated in panel (a) with monolayer, bilayer and trilayer graphene, under light illumination at $\lambda=10 \mu \mathrm{m} . \mathrm{V}_{\mathrm{DS}}=2 \mathrm{mV}$ and $\mathrm{V}_{\mathrm{G}}=80 \mathrm{~V}$. Reproduced with permission from X. C. Yu et al., Nat. Commun. 9, 4299 (2018). Licensed under a Creative Commons Attribution (CCBY_4.0) license. (d-f) MIR photodetection by coupling graphene with narrow-bandgap topological insulator $\mathrm{Bi}_{2} \mathrm{Se}_{3} .{ }^{45}$ (d) Schematic of graphene- $\mathrm{Bi}_{2} \mathrm{Se}_{3}$ heterostructure PD and electrical measurement circuit. (e) Illustration of band diagram and transfer process of photocarriers under light illumination. (f) Transfer characteristics measured with (red curve) and without (blue curve) MIR illumination at $\lambda=3.5 \mu \mathrm{m}$ (illumination power: $50 \mu \mathrm{W}$ ). Source-drain bias voltage is $0.5 \mathrm{~V}$. Reproduced with permission from J. Kim et al., ACS Photonics 4, 482 (2017). Copyright 2017 American Chemical Society. (g-i) MIR photodetection with graphene PD consisting of graphene double-layer sandwiching a tunneling barrier ( $\sim \mathrm{nm}$ thick intrinsic silicon layer). ${ }^{46}$ (g) Schematic of graphene double-layer heterostructure PD. (h) Band diagram illustration of PD and depiction of photocarrier transport. (i) Gate-dependent photocurrent measured under different illumination powers at $\lambda=3.2 \mu \mathrm{m} . \mathrm{V}_{\mathrm{DS}}=1.5 \mathrm{~V}$. Reproduced with permission from C. H. Liu et al., Nat. Nanotechnol. 9, 273 (2014).

\section{Integration with plasmonic nanoantennas/nanoparticles/engineered electrodes}

Plasmonic nanostructures supporting propagating/localized surface plasmon polaritons are widely used to enhance light-matter interactions, as they can produce strong electric fields confined to subwavelength regions. This property is particularly attractive for boosting the light-graphene interaction and therefore improving the optical absorption in graphene, ${ }^{59}$ because the low intrinsic absorption of graphene $(<2.3 \%)^{60}$ is a major limiting factor for the achievable responsivity in graphene PDs. When integrated with graphene, plasmonic nanostructures can also act as nanoelectrodes that help carrier collection. This approach also facilitates fast detector response as the responsivity enhancement does not come at the expense of increased carrier lifetime. Figure 6 shows 
several examples that demonstrate high responsivity in graphene MIR PDs by integration with plasmonic nanoantennas, ${ }^{39,47,50}$ plasmonic nanoparticles ${ }^{48}$ and engineered electrodes ${ }^{49}$. Figures 6 (a) and 6(b) shows a work in which plasmonic end-to-end linear antennas are employed to enhance both the optical absorption and carrier collection efficiency in graphene. ${ }^{39}$ The device working principle is as follows. The linear antennas collect the incident light and concentrate it into the narrow gaps $(\sim 100 \mathrm{~nm})$ between adjacent antennas, whereby the optical absorption in the graphene is boosted (to be up to $\sim 10 \%$, according to simulations) due to the enhanced electric fields. The photogenerated carriers are then efficiently collected by the nanoantennas as photocurrent. In this device, a responsivity of $0.4 \mathrm{~V} / \mathrm{W}$ is obtained at room temperature at a wavelength of $4.45 \mu \mathrm{m}$ under a bias current of $4 \mathrm{~mA}$ (Fig. 6(b)), which represents an improvement of more than 200 times over reference devices without the antennas $(<2 \mathrm{mV} / \mathrm{W})$. A similar configuration is employed in another work (Fig. 6(c)) but with T-shaped antennas instead of linear antennas. ${ }^{50}$ In that work, polarization-sensitive photodetection is reported via a bulk photovoltaic effect assisted by the non-centrosymmetric metallic nanoantennas. The authors explain the mechanism to be as follows. Hot photocarriers in graphene gain momentum via both a gradient in the Seebeck coefficient and a conductance guidance due to the presence of the metallic nanoantennas. The result is a shift current which is nonlocal and directional. A room-temperature and zero-bias responsivity of $16.6 \mathrm{~mA} / \mathrm{W}$ is observed under uniform illumination at a wavelength of $4 \mu \mathrm{m}$. Due to the low symmetry of the employed nanoantennas, ${ }^{39,50}$ the response of these detectors is strongly polarization dependent. In another work (Figs. 6(d)-6(f)), ${ }^{47}$ JerusalemCross shaped antennas (JC-antennas) are employed instead of linear antennas to further increase the light absorption in graphene and achieve a polarization-independent response (Fig. 6(f)). The highly symmetric shape of the JC-antennas and narrower antenna gaps $(\sim 40 \mathrm{~nm})$ render a graphene light absorption of $16.4 \%$, which is $64 \%$ and $167 \%$ larger than that occurring in the linear antenna configuration discussed above, ${ }^{39}$ for linearly-polarized and unpolarized light respectively. As a result of the enhanced graphene absorption and carrier collection enabled by the JC-antennas, a room- 
temperature responsivity of $4.6 \mathrm{~mA} / \mathrm{W}$ (which corresponds to $14.5 \mathrm{~V} / \mathrm{W}$ ) is obtained in the MIR, under a source-drain bias voltage of $0.1 \mathrm{~V}$. This work also achieved a fast response, with a measured rise time of $\sim 3 \mathrm{~ns}$. The absorption in graphene can also be enhanced using plasmonic silicon quantum dots (Si QDs) doped with boron (B), as reported in a work shown in Figs. 6(g)-6(i). ${ }^{48}$ The localized surface plasmon resonance (LSPR) of the B-doped Si QDs enhances the MIR absorption of graphene (Fig. 6(h)), enabling sensitive photodetection with a measured responsivity of around $44.9 \mathrm{~A} / \mathrm{W}$ at 77 $\mathrm{K}$ at a wavelength of $3 \mu \mathrm{m}\left(\mathrm{V}_{\mathrm{DS}}=1 \mathrm{~V}\right)$, as shown in Fig. 6(i). The use of plasmonic nanoantennas can enhance responsivity while maintaining fast response, but also can limit the spectral bandwidth over which the detector operates, due to the resonant nature of the antenna plasmons. By employing a novel carrier collection strategy using engineered nanoelectrodes with graphene nano-strips, researchers demonstrate simultaneous broadband and ultra-fast photodetection with high responsivity (Figs. 6(j) and 6(k)). ${ }^{49}$ These nanoelectrodes enable a modest and broadband optical absorption enhancement in the graphene, and help to collect the photocarriers efficiently before their recombination. The authors report that this device shows a sensitive and ultra-broadband photodetection from the visible to the MIR, with operation frequencies exceeding $50 \mathrm{GHz}$. Roomtemperature MIR responsivities of around 2-11.5 A/W are observed at wavelengths from 3-20 $\mu \mathrm{m}$, under a source-drain bias voltage of $20 \mathrm{mV}$ (Fig. 6(k)). 

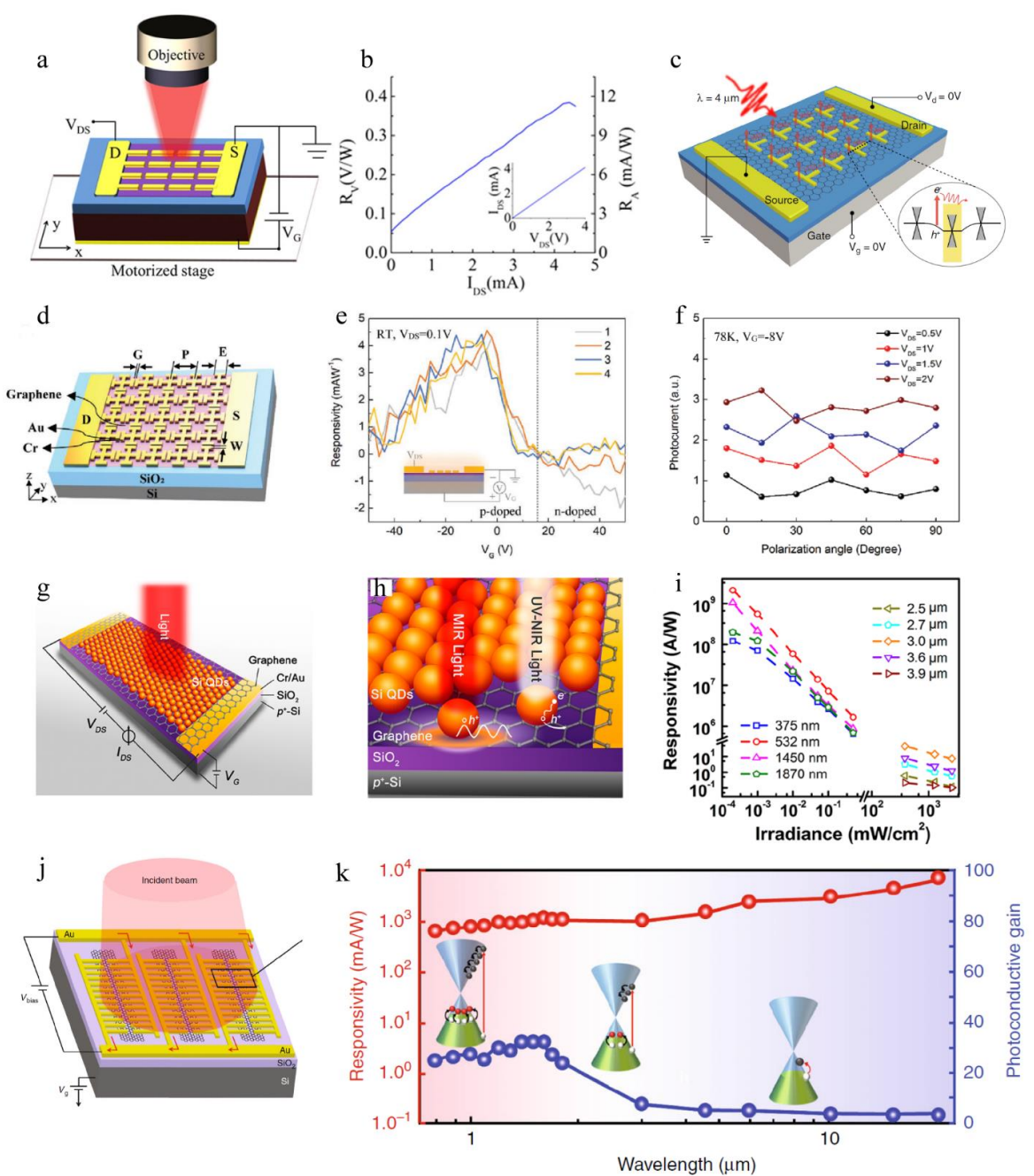

FIG. 6. Integration with plasmonic nanoantennas $\left((a-b),{ }^{39} c,{ }^{50}(d-f)^{47}\right) /$ nanoparticles $\left.(g-i)\right)^{48} /$ engineered electrodes $(\mathrm{j}, \mathrm{k})^{49}$. (a) Schematic of graphene PD integrated with linear nanoantennas on a $\mathrm{SiO}_{2} / \mathrm{Si}_{\text {substrate. }}{ }^{39}$ (b) Bias-dependent responsivity measured at room temperature under gate voltage of $4 \mathrm{~V}$. Inset: $\mathrm{V}_{\mathrm{DS}}-\mathrm{I}_{\mathrm{DS}}$ plot of same detector without illumination (i.e. in dark). Reproduced with permission from Y. Yao et al., Nano Lett 14, 3749 (2014). Copyright 2014 American Chemical Society. (c) Schematic of metasurface-mediated graphene photodetector, which consists of non-centrosymmetric sub-wavelength metallic nanoantennas on top 
of graphene flakes on a $\mathrm{SiO}_{2} / \mathrm{Si}$ substrate. ${ }^{50}$ Inset: Illustration of band diagram of graphene and directional carrier transport. Reproduced with permission from J. X. Wei et al., Nat. Commun. 11, 6404 (2020). Licensed under a Creative Commons Attribution (CC-BY_4.0) license. (d) Schematic of polarization-independent graphene PD integrated with Jerusalem-Cross nanoantennas on a $\mathrm{SiO}_{2} / \mathrm{Si}$ substrate. ${ }^{45}$ (e) Room-temperature responsivity as a function of gate voltage measured under source-drain bias voltage of $0.1 \mathrm{~V}$ in MIR. Inset shows measurement circuit. (f) Polarization-resolved photocurrent measured at $78 \mathrm{~K}$ under different sourcedrain bias voltages, with fixed gate voltage of $-8 \mathrm{~V}$. Reproduced with permission from M. Ye et al. Adv. Opt. Mater. 9, 2001854 (2021). (g) Schematic of hybrid phototransistor based on boron-doped Si QDs and graphene. $^{48}(\mathrm{~h})$ Illustration of roles of Si QDs in photodetection in both MIR and ultraviolet to near-infrared (UV-to-NIR) regions. In MIR region, strong near field associated with LSPR of B-doped Si QDs enhances light absorption in graphene, leading to sensitive detection of MIR light. (i) Measured responsivity as a function of laser irradiance at different laser wavelengths at $\mathrm{V}_{\mathrm{G}}=0 \mathrm{~V}$ and $\mathrm{V}_{\mathrm{DS}}=1 \mathrm{~V}$. Reproduced with permission from Z. Y. Ni et al., ACS Nano 11, 9854 (2017). Copyright 2017 American Chemical Society. (j) Schematic of PD based on graphene nano-stripes and engineered nanoelectrodes. ${ }^{49}(\mathrm{k})$ Measured responsivity (red) and photoconductive gain (blue) at optical power of $2.5 \mu \mathrm{W}$, gate voltage of $22 \mathrm{~V}$, and bias voltage of 20 mV. Reproduced with permission from S. Cakmakyapan et al., Light Sci. Appl. 7, 20 (2018). Licensed under a Creative Commons Attribution (CC-BY_4.0) license.

\section{Integration with silicon waveguide}

The use of in-plane evanescent light absorption in graphene is demonstrated to enable efficient MIR photodetection in a graphene/silicon-heterostructure waveguide PD on a silicon-on-insulator (SOI) substrate, as shown in Fig. $7 .^{52}$ The graphene/ silicon-heterostructure photodiode is formed by integrating graphene onto a silicon optical waveguide on SOI (Fig. 7(a)). A focusing subwavelength grating is used to couple light from an optical fiber into the silicon/graphene waveguide. In this device, the in-plane evanescent light (rather than normally-incident light) propagating parallel to the graphene sheet is absorbed via indirect interband transition, resulting in a high responsivity of $0.13 \mathrm{~A} / \mathrm{W}$ at a 
bias voltage of $-1.5 \mathrm{~V}$ for $\lambda=2.75 \mu \mathrm{m}$ excitation at room temperature. The formation of a heterostructure between the graphene and silicon waveguide leads to a strongly bias-polarity dependent photoresponse, as can be seen in Figs. 7(b) and 7(c). The potential barrier at the graphene/silicon junction interface facilitates charge separation for reverse bias (graphene is biased positive with respect to silicon) and blocks electron transport from graphene to silicon for forward bias.

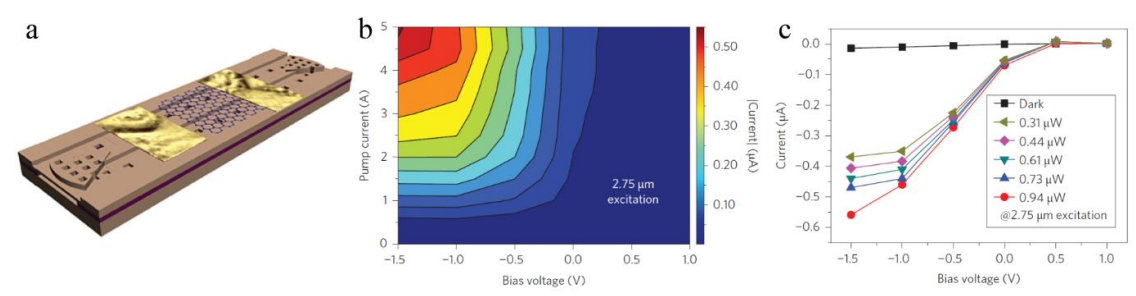

FIG. 7. Integration with silicon waveguide on a silicon-on-insulator (SOI). ${ }^{52}$ (a) Schematic of a graphene/silicon-heterostructure waveguide PD. (b) Current as a function of pump current of excitation laser (wavelength: $2.75 \mu \mathrm{m}$ ) and bias voltage measured at room temperature. (c) Current as a function of bias voltage under dark and light conditions for different incident light powers. Reproduced with permission from X. M. Wang et al., Nat. Photonics 7, 888 (2013).

\section{E. Enhanced PTE effect with asymmetric plasmons}

The plasmon-assisted PTE effect has been employed for room-temperature MIR photodetection, as discussed in section II and Figs. 1(d)-1(i). However, in that work, ${ }^{30}$ the temperature increase of the charge carriers is only $1.72 \mathrm{~K}$, which results in a modest responsivity of $16 \mathrm{~mA} / \mathrm{W}$ at room temperature. For further improvement of the detector responsivity, the generation of larger temperature gradients across the channel would be desirable. A typical example towards achieving this goal is shown in Fig. 8, where the plasmon-assisted PTE effect is enhanced by engineering an asymmetric electronic environment of the plasmonically excited hot carriers on the graphene. ${ }^{53}$ The 
PD (Fig. 8(a)) consists of a graphene field effect transistor with an optical cavity on top. Half of the graphene channel is patterned with a hexagonal array of holes so that upon illumination, graphene plasmons are selectively excited in the patterned section of the graphene. As a result, hot carriers are excited asymmetrically across the channel, giving rise to an enhanced temperature gradient across the channel $(4.7 \mathrm{~K}$ under incident power of $153 \mathrm{nW}$ at an excitation wavelength of $8.15 \mu \mathrm{m}$, top panel of Fig. 8(b)) and thereby yielding an enhanced PTE voltage (0.44 mV, bottom panel of Fig. 8(b)). Due to the large temperature gradient, a strong room-temperature PTE photoresponse is obtained at longwave infrared (LWIR) region $(8-12 \mu \mathrm{m})$, with a measured maximum responsivity of $2900 \mathrm{~V} / \mathrm{W}$ at a source-drain bias voltage of $0.9 \mathrm{~V}$ (Fig. 8(c), red curve). A response time of $\sim 100 \mathrm{~ns}$ is found by temporally modulating the electrical bias signal (under constant LWIR illumination).
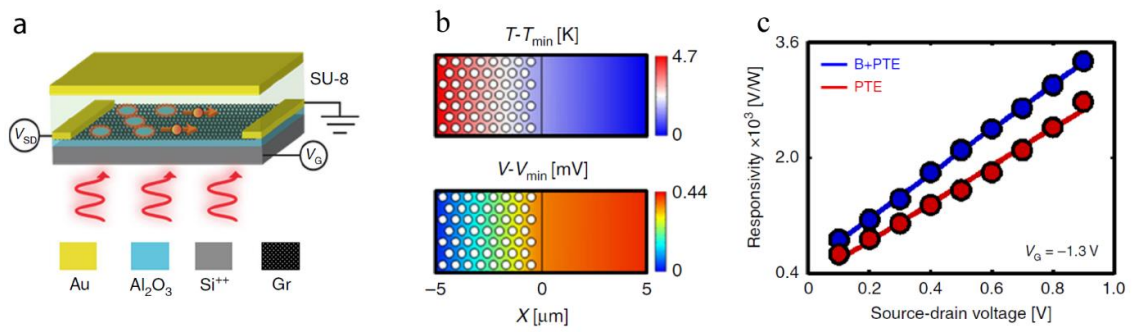

FIG. 8. Asymmetric plasmon-assisted PTE effect for responsivity enhancement of graphene PD. ${ }^{53}$ (a) Schematic of a graphene PD with an asymmetrically nanopatterned graphene channel. (b) Simulated temperature (top) and PTE voltage (bottom) profiles of graphene PD. Graphene Fermi level $\mathrm{E}_{\mathrm{F}}=-0.85 \mathrm{eV}$, illumination wavelength $\lambda=8.15 \mu \mathrm{m}$, illumination power $\mathrm{P}=153 \mathrm{nW}$, source-drain bias $\mathrm{V}_{\mathrm{SD}}=0.9 \mathrm{~V}$. (c) $\mathrm{MIR}$ responsivity measured as a function of source-drain bias voltage at a gate voltage of $-1.3 \mathrm{~V}$. Blue curve corresponds to total responsivity. Red curve corresponds to responsivity due to PTE contribution from asymmetric hot-carrier generation. Reproduced with permission from A. Safaei et al., Nat. Commun.10, 1 (2019). Licensed under a Creative Commons Attribution (CC-BY_4.0) license. 


\section{F. Enhanced PTE effect in graphene p-n junction by coupling plasmonic antenna to hyperbolic phonon-polaritons (HPPs) in hexagonal boron nitride (h-BN)}

Graphene PDs based on the PTE effect in graphene $\mathrm{p}-\mathrm{n}$ junctions have drawbacks such as small active area (limited by the cooling length of hot carriers $(0.5-1 \mu \mathrm{m})^{20,61}$ ) and weak absorption, which generally lead to a low detector responsivity. To improve the responsivity of this type of graphene PDs, hyperbolic phonon-polaritons (HPPs) in hexagonal boron nitride (h-BN) have been exploited to focus the incident MIR light into the graphene $\mathrm{p}-\mathrm{n}$ junction and boost the optical absorption. ${ }^{54,55}$ HPPs in h-BN can concentrate MIR light into deep subwavelength scales. Figure 9 shows an example in which sensitive and fast MIR photodetection is demonstrated by coupling the plasmonic resonances with HPPs in h-BN to achieve highly concentrated fields in a graphene $\mathrm{p}-\mathrm{n}$ junction detector. ${ }^{55}$ The device is fabricated by embedding h-BN and graphene within metallic antennas that comprise a top bowtie antenna and a bottom H-shaped antenna (which also serves as a split-gate), as illustrated in Figs. 9(a) and 9(b). Highly concentrated fields within the graphene p-n junction area result from the coupling of the LSPRs of the top bowtie antenna (for TM-polarization, Fig. 9(c)) or the bottom H-shaped antenna (for TE-polarization, Fig. 9(d)) to the HPPs mode in h-BN (upper reststrahlen band (RB), range: $\lambda=6-7 \mu \mathrm{m}$ ) and subsequent interference within the h-BN waveguide. The resultant large temperature gradient then produces a strong PTE photoresponse when voltages of opposite signs are applied to the two gates. The PTE driven photodetection mechanism can be verified by the six-fold photocurrent pattern as shown in Fig. 9(e), which is also observed in other studies in the MIR range. ${ }^{54,62}$ High responsivities of $15 \mathrm{~mA} / \mathrm{W}$ and $22 \mathrm{~mA} / \mathrm{W}$ are demonstrated for TM- (Fig. 9(f)) and TE- (Fig. 9(g)) polarizations, respectively, within the h-BN RB. 

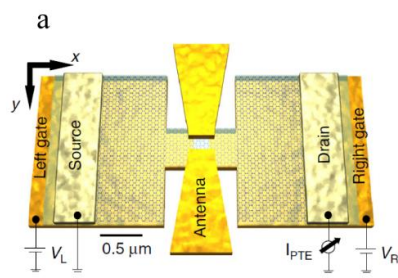

$\mathrm{b}$
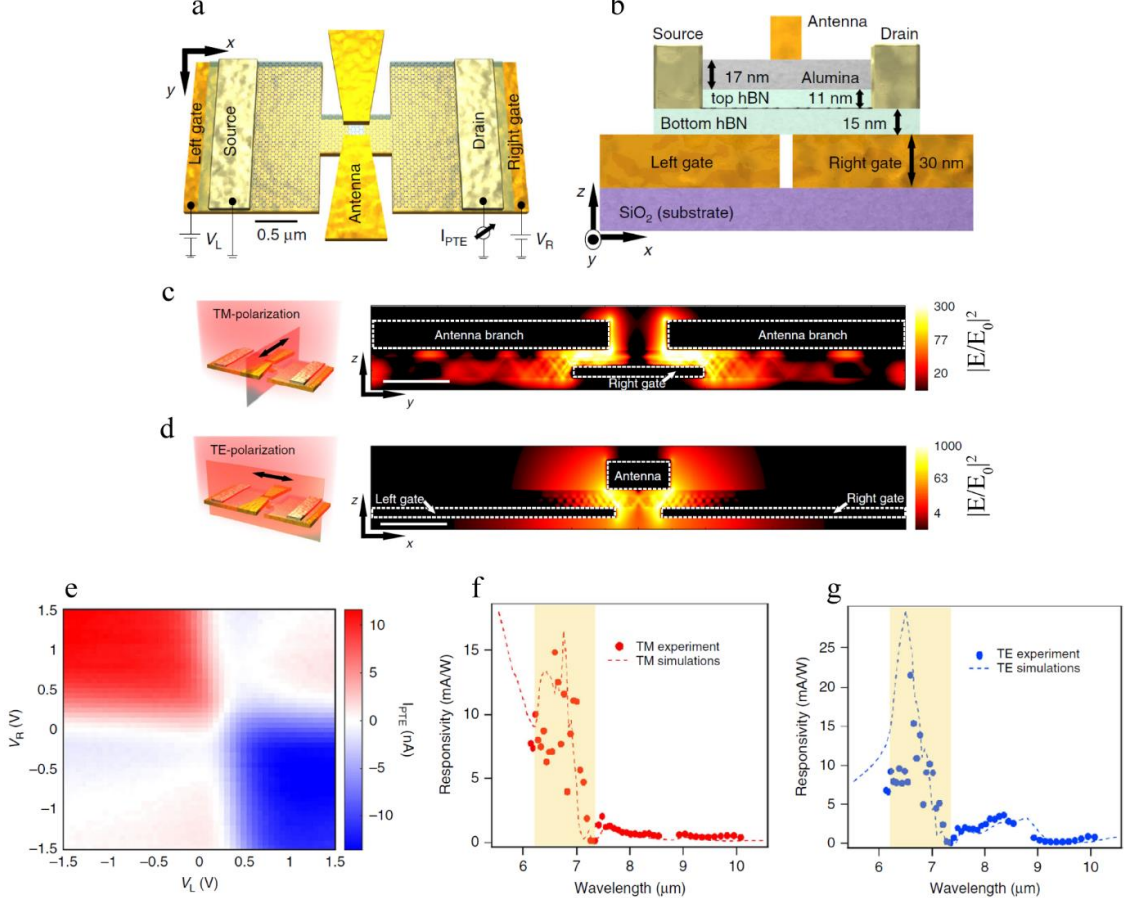

FIG. 9. Enhancing PTE effect in graphene $\mathrm{p}-\mathrm{n}$ junction by coupling plasmonic antenna to hyperbolic phononpolaritons (HPPs) in hexagonal boron nitride (h-BN) ${ }^{55}$ (a) Schematic of PD that consists of H-shaped resonant bottom gates and top bowtie antennas, with an h-BN-encapsulated $\mathrm{H}$-shaped graphene channel. (b) Side-view illustration of device structure described in (a). (c) Cross section view of simulated normalized total electric field intensity along main axis of bowtie antennas when light is polarized parallel to bowtie antennas (TMpolarization). White scale bar: $250 \mathrm{~nm}$. (d) Same as (c) but for light polarization parallel to bottom gates. (e) Photocurrent map as a function of two gate voltages, measured at $\lambda=6.6 \mu \mathrm{m}$. (f-g) Measured (dots) and predicted (dashed lines) responsivity spectra for TM- (f) and TE- (g) polarizations. Left and right applied gate voltages are $0.5 \mathrm{~V}$ and $-0.5 \mathrm{~V}$, respectively. Highlighted regions correspond to h-BN $\mathrm{RB}(\lambda=6.2-7.3 \mu \mathrm{m})$. Adapted with permission from S. Castilla et al., Nat. Commun. 11, 4872 (2020). Licensed under a Creative Commons Attribution (CC-BY_4.0) license. 


\section{G. Small-twist-angle bilayer graphene}

Small-twist-angle $\left(<2^{\circ}\right)$ bilayer graphene $(\mathrm{TBG})$ is an emerging material platform which possesses exciting physical properties that has inspired many research efforts in the past few years. ${ }^{63}$ Recently, this novel material platform has been used for the efficient detection of MIR light. ${ }^{56}$ The device architecture (Fig. 10(a)) consists of a h-BN/TBG/h-BN stack on a $\mathrm{SiO}_{2} / \mathrm{Si}$ substrate. Unlike monolayer graphene, Brillouin zone folding in the TBG (twist angle of $1.81^{\circ}$ ) leads to the formation of a superlattice bandgap and substantial modification to the density of states, which is responsible for the realization of efficient MIR detection. This leads to the three peaks appearing in the measured gate-dependent channel resistance as shown in Fig. 10(b). One peak occurs at the charge-neutrality point of the TBG. The other two peaks occur at gate voltages of $\pm 43.5 \mathrm{~V}$ and are caused by the formation of superlattice bands above and below the lowest moiré Dirac bands (inset of Fig. 10(b)). These two peaks are observed when the Fermi level is tuned to the center of the superlattice bandgap, whereby the carrier density in the channel is minimized. Efficient photodetection is realized at MIR wavelengths when the Fermi level is tuned to the center of the superlattice bandgap, with a measured responsivity of $26 \mathrm{~mA} / \mathrm{W}$ at $83 \mathrm{~K}$ under illumination at $\lambda=12 \mu \mathrm{m}\left(\mathrm{V}_{\mathrm{DS}}=200 \mathrm{mV}\right)$, as shown in Fig. 10(c). The efficient photodetection can be ascribed to the increased channel conductivity of the TBG compared to un-twisted bilayer graphene (Fig. 10(d)) and the formation of a bandgap that allows absorption of MIR light. The authors conclude that the detection mechanism is mainly a bolometric effect, a finding that is inferred from the polarity changes in the responsivity curves (Fig. 10(c)). The bolometric effect usually results in a negative photocurrent for metals and superconductors, but a positive photocurrent in semiconductors. The behaviors of the measured photocurrent polarities are found to be consistent with the temperature-dependent transport characterizations under the assumption of a bolometric mechanism. As can be seen in Fig. 10(e), when the Fermi level is close to the superlattice bandgap, the TBG resistance decreases as the temperature increases, featuring a semiconductor behavior. While when the Fermi level is away from the superlattice bandgap, the TBG 
resistance increases as the temperature increases, which indicates a metallic behavior of the TBG channel. The extracted gate dependence of the temperature coefficient of conductance (TCC) in the inset of Fig. 10(e) resembles that of the photoresponse in Fig. 10(c), indicating that the photodetection is driven by the bolometric effect.
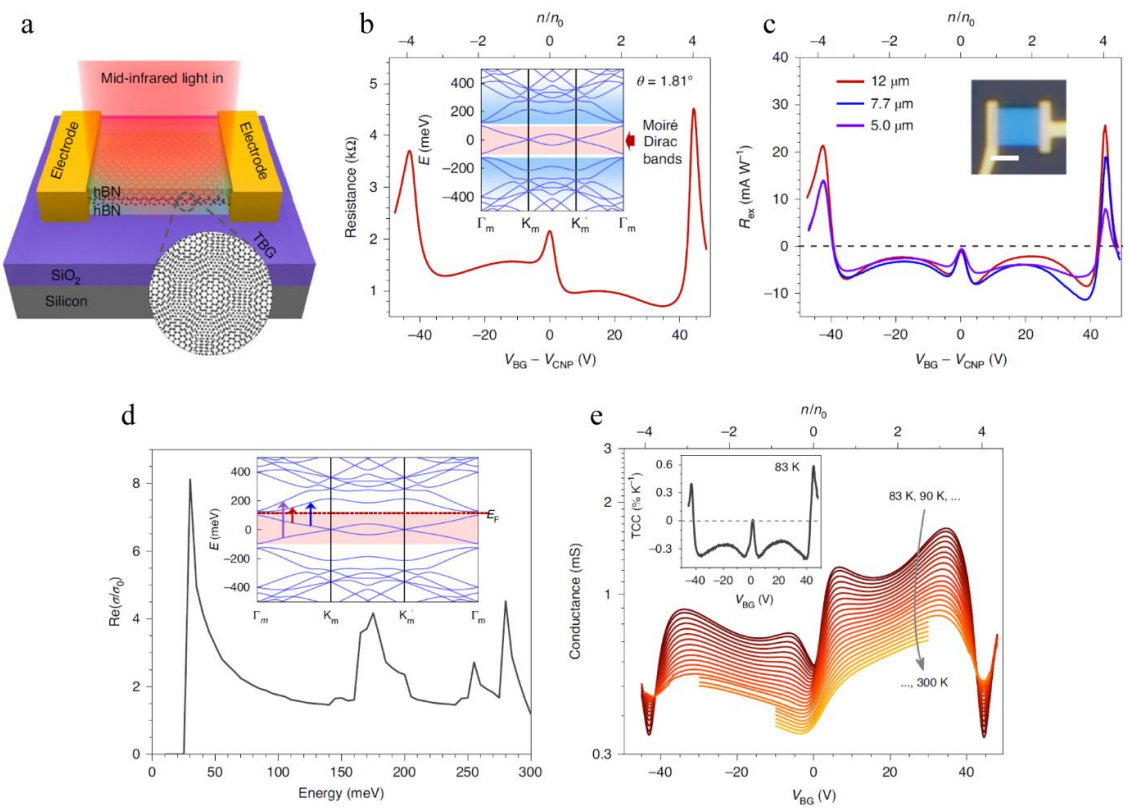

FIG. 10. Efficient MIR detection in small-twist-angle bilayer graphene (TBG) PD. ${ }^{56}$ (a) Schematic of detector consisting of h-BN/TBG/h-BN stack on $\mathrm{SiO}_{2} / \mathrm{Si}$ substrate. Twist angle of TBG in device is $1.81^{\circ}$. (b) Gatedependent channel resistance measured at $83 \mathrm{~K}$, with source-drain bias of $10 \mathrm{mV}$. Upper $\mathrm{x}$-axis shows electron filling states. Inset shows calculated band structure of $1.81^{\circ} \mathrm{TBG}$. (c) Gate-dependent responsivity of device measured at $83 \mathrm{~K}$ for MIR light at $\lambda=5,7.7$ and $12 \mu \mathrm{m}$. Source-drain bias voltage is kept at $200 \mathrm{mV}$. Inset shows optical image of $1.81^{\circ} \mathrm{TBG}$ device. Scale bar is $2 \mu \mathrm{m}$. (d) Calculated dynamical conductivity (real part) spectrum of $1.81^{\circ} \mathrm{TBG}$ for Fermi level at the middle of superlattice bandgap in electron branch. Arrows in inset show possible interband transitions for MIR illuminations at $\lambda=5,7.7$ and $12 \mu \mathrm{m}$. (e) Gate-dependent conductance measured at different temperatures. Inset shows calculated temperature coefficient of 
conductance (TCC) at $83 \mathrm{~K}$, which is derived from temperature-dependent conductance. Reproduced with permission from B. C. Deng et al., Nat. Photonics 14, 549 (2020).

To put things into perspective, key figures-of-merit of the graphene MIR PDs discussed in this article that employ different responsivity enhancement approaches are presented in Table II. To put them in the context of the topic of this review, the figures-of-merit shown are for the MIR spectral region. However, it should be noted that in general graphene PDs can operate over broad spectral ranges, though the responsivity enhancement might be spectrally selective in some cases, for example when plasmonic nanostructures are integrated, when asymmetric graphene plasmons are employed, etc.

TABLE II. Key figures-of-merit of detectors employing various responsivity enhancement methods.

\begin{tabular}{|c|c|c|c|c|c|c|}
\hline $\begin{array}{l}\text { Enhancement } \\
\text { method }\end{array}$ & Device description & Wavelength & Responsivity & $\begin{array}{c}\text { Bias } \\
\text { voltage }\end{array}$ & $\begin{array}{c}\text { Response } \\
\text { time }\end{array}$ & Ref. \\
\hline $\begin{array}{l}\text { Band structure } \\
\text { engineering }\end{array}$ & Graphene QDs & $\sim 10 \mu \mathrm{m}$ & $0.4 \mathrm{~A} / \mathrm{W}$ & $0.02 \mathrm{~V}$ & $\sim 10-100 \mathrm{~s}$ & 42 \\
\hline \multirow{3}{*}{ Photogating effect } & Hybrid graphene $/ \mathrm{Ti}_{2} \mathrm{O}_{3}$ & $10 \mu \mathrm{m}$ & $300 \mathrm{~A} / \mathrm{W}$ & $0.002 \mathrm{~V}$ & $1-3 \mathrm{~ms}$ & 44 \\
\hline & $\begin{array}{l}\text { Graphene/ } \mathrm{Bi}_{2} \mathrm{Se}_{3} \\
\text { heterostructure }\end{array}$ & $3.5 \mu \mathrm{m}$ & $1.97 \mathrm{~A} / \mathrm{W}$ & $0.5 \mathrm{~V}$ & $4 \mu \mathrm{s}$ & 45 \\
\hline & $\begin{array}{l}\text { Graphene double-layer } \\
\text { heterostructure }\end{array}$ & $3.2 \mu \mathrm{m}$ & $1.1 \mathrm{~A} / \mathrm{W}$ & $1.5 \mathrm{~V}$ & $1-100 \mathrm{~ms}$ & 46 \\
\hline \multirow{5}{*}{$\begin{array}{l}\text { Integration with } \\
\text { plasmonic } \\
\text { nanostructure }\end{array}$} & Linear rod antennas & $4.45 \mu \mathrm{m}$ & $0.4 \mathrm{~V} / \mathrm{W}$ & $4 \mathrm{~V}$ & $\sim 63 \mathrm{~ns}$ & 39 \\
\hline & T-shaped antennas & $4 \mu \mathrm{m}$ & $16.6 \mathrm{~mA} / \mathrm{W}$ & Zero bias & $<100 \mu \mathrm{s}$ & 50 \\
\hline & Jerusalem-Cross antennas & $3-5 \mu \mathrm{m}$ & $14.5 \mathrm{~V} / \mathrm{W}$ & $0.1 \mathrm{~V}$ & $\sim 3 \mathrm{~ns}$ & 47 \\
\hline & Boron-doped Si QDs & $3 \mu \mathrm{m}$ & $44.9 \mathrm{~A} / \mathrm{W}$ & $1 \mathrm{~V}$ & $3.4-9 \mathrm{~s}$ & 48 \\
\hline & Engineered electrodes & $3-20 \mu \mathrm{m}$ & $2-11.5 \mathrm{~A} / \mathrm{W}$ & $0.02 \mathrm{~V}$ & $<20 \mathrm{ps}$ & 49 \\
\hline $\begin{array}{l}\text { Integration with } \\
\text { waveguide }\end{array}$ & $\begin{array}{l}\text { Graphene/silicon- } \\
\text { heterostructure waveguide }\end{array}$ & $2.75 \mu \mathrm{m}$ & $0.13 \mathrm{~A} / \mathrm{W}$ & $-1.5 \mathrm{~V}$ & I & 52 \\
\hline $\begin{array}{l}\text { Use of asymmetric } \\
\text { plasmon }\end{array}$ & $\begin{array}{l}\text { Asymmetrically patterned } \\
\text { graphene channel }\end{array}$ & $8-12 \mu \mathrm{m}$ & $2900 \mathrm{~V} / \mathrm{W}$ & $0.9 \mathrm{~V}$ & $\sim 100 \mathrm{~ns}$ & 53 \\
\hline \multirow{2}{*}{$\begin{array}{l}\text { Coupling LSPRs to } \\
\text { HPPs }\end{array}$} & $\begin{array}{l}\text { Graphene } \mathrm{p}-\mathrm{n} \text { junction } \\
\text { integrated with split-gate } \\
\text { and h-BN }\end{array}$ & $6.6 \mu \mathrm{m}$ & $1 \mathrm{~V} / \mathrm{W}$ & Zero bias & I & 54 \\
\hline & $\begin{array}{l}\text { Graphene p-n junction } \\
\text { integrated with bowtie } \\
\text { antenna and h-BN }\end{array}$ & $6.2-7.3 \mu \mathrm{m}$ & $15-22 \mathrm{~mA} / \mathrm{W}$ & Zero bias & $17 \mathrm{~ns}$ & 55 \\
\hline \multirow{3}{*}{$\begin{array}{l}\text { Use of small twist- } \\
\text { angle bilayer } \\
\text { graphene }\end{array}$} & & $5.0 \mu \mathrm{m}$ & $14 \mathrm{~mA} / \mathrm{W}$ & & & \\
\hline & $\begin{array}{l}\text { Small-twist-angle bilayer } \\
\text { graphene }\end{array}$ & $7.7 \mu \mathrm{m}$ & $18 \mathrm{~mA} / \mathrm{W}$ & $0.2 \mathrm{~V}$ & I & 56 \\
\hline & & $12 \mu \mathrm{m}$ & $26 \mathrm{~mA} / \mathrm{W}$ & & & \\
\hline
\end{tabular}




\section{OUTLOOK AND CONCLUSIONS}

Over the past decade, much progress has been made in improving the performance of MIR graphene-based PDs in aspects that include high responsivity, high speed, broadband spectral response and room-temperature operation, by employing metamaterials and related concepts as discussed in this review. Indeed, some of the developed graphene MIR PDs show responsivities (e.g. $300 \mathrm{~A} / \mathrm{W}$ ) and/or operation speeds (e.g. > $50 \mathrm{GHz}$ ) that are on par with if not exceeding that of the state-of-the-art commercial PDs. Ultra-broadband spectral response from the visible to the MIR has also been realized in a single graphene PD device, which is unmatched by existing PDs based on other material systems. ${ }^{49}$ It should also be pointed out that in contrast to the complex and costly epitaxial growth of conventional narrow-bandgap semiconductors, many of the reported highperformance graphene MIR PDs are based on graphene grown by CVD, a method that has been commercialized and offers low cost and scalability. It might be the case that the graphene quality is degraded during fabrication processes such as when it is transferred from the growth substrate to the device substrate, lithography, lift-off etc. Such degradation could potentially be alleviated by improvement of the nanofabrication techniques and/or by directly growing graphene on the target substrate via CVD. Despite the progress that has been achieved, there are still much room for further improvements with modifications/optimizations to existing approaches or by exploring novel methods that take advantage of the unique optical and electronic properties of graphene. For example, while the plasmon-assisted PTE effect in graphene has been utilized for MIR photodetection, the detector responsivity is often low due to the modest optical absorption $\left(<10 \%^{30}\right)$ and temperature increase of charge carriers $\left(\sim 1.72 \mathrm{~K}^{30}\right)$ in graphene. Although the engineering of asymmetric graphene plasmons is able to result in a larger temperature increase $(\sim 4.7 \mathrm{~K}),{ }^{53}$ it would be interesting to see whether asymmetrically excited metal plasmons could also be employed in a similar way to achieve even greater increases in absorption and temperature, by leveraging the much stronger electric fields associated with metal plasmons. In addition, although graphene MIR plasmons have been exploited 
to resonantly enhance absorption and demonstrate room-temperature MIR photodetection, its appealing gate-tunable property has not been seriously investigated for realizing electrically tunable spectrally-selective photoresponse, which could present opportunities for developing miniaturized MIR spectrometers. We discuss this further below. One major challenge that remains for most current graphene MIR PDs is the large dark current, which often occurs in the graphene channel in photoconductor and phototransistor configurations due to the semi-metallic nature of graphene. This imposes a minimum shot noise-limited noise level and thus limits the maximum achievable detectivity. One way to reduce the dark current could be to form heterostructures between graphene and other two-dimensional (2D) materials, where the existence of interface potential barrier can suppress the dark current significantly. Heterostructures between graphene and other 2D materials can be readily formed by stacking them on top of one another, with lattice matching not being problematic due to the self-terminating lattices of 2D materials. Currently, such heterostructures have mainly been demonstrated for photodetection in the visible-NIR spectral region, using graphene and 2D transition metal dichalcogenides (TMDs) such as $\mathrm{MoS}_{2}{ }^{64}$ and $\mathrm{WS}_{2}{ }^{65}$. With the emergence of the 2D black phosphorus (bP), whose bandgap can be tuned via atomic or electrostatic doping to allow efficient MIR absorption, ${ }^{66}$ it could be expected that graphene-bP heterostructure devices hold good promise for realizing low dark current and sensitive photodetection in the MIR region. Such devices would benefit from the strong MIR absorption in bP, effective dark current suppression and efficient charge separation at the heterojunctions. Last but not least, the new platform of small-twist-angle bilayer graphene, with its unique electronic properties, is just beginning to be investigated for MIR photodetection. Much effort is needed for further improving the detector performance. The approaches developed for single layer graphene-based detectors could in principle be employed to improve the performance of detectors based on small-twist-angle bilayer graphene.

As the performance of graphene MIR PDs has been and is expected to continue to be improved significantly, a wide range of emerging applications will become increasingly practical, by leveraging 
the unique properties of graphene such as flexibility, transparency, CMOS integrability and the possibility for cost effective manufacturing. These applications include graphene-integrated biosensors, imaging sensors and spectrometers, to name a few. These devices could be made bendable and portable, and could be integrated with Si-CMOS read-out circuits, paving the way for the realization of next generation optoelectronic devices. Among these applications, graphene MIR PDs provide great opportunities for developing chip-scale spectrometers, also known as microspectrometers, working in the MIR region. Recent years have witnessed a growing trend in the miniaturization of spectrometers, motivated by their potential applications including lab-on-a-chip system, drones, and wearable devices. Microspectrometers have advantages over conventional systems such as diffraction grating-based spectrometers and Michelson interferometer-based Fourier transform infrared spectrometers (FTIR). These conventional benchtop systems are often heavy, bulky, expensive and require precise alignment of optical components and the incoming beam. Microspectrometers can have volumes and masses on the order of several cubic centimeters and grams, respectively. Microspectrometers are also invulnerable to misalignment issues for there are no moving components. Microspectrometers have been recently demonstrated in which the combination of the responsivity spectra of the detector and a reconstruction algorithm enables spectral reconstruction of the incoming light. ${ }^{67,68}$ In these microspectrometers, spectral filtering and photocurrent generation occur within the same nanostructure, giving rise to tailored responsivities of the detector pixels. The measured photocurrent from each pixel, together with pre-calibrated response functions from the pixels, are then processed in a reconstruction algorithm to reconstruct the spectrum of incident light. Figure 11(a) shows a microspectrometer chip based on structurally colored silicon nanowire arrays. ${ }^{68}$ Each colored square is a uniform array of nanowires of a specific radius. The latter controls the absorption of light through the supported leaky waveguide modes, thereby tailoring the responsivity spectrum of each pixel. Figure 11(b) shows an SEM image of the etched nanowires of a pixel. Each nanowire consists of a vertical PIN photodiode that contains the following layers (top to 
bottom): $\mathrm{p}+$ layer (200 nm thick), lightly doped silicon ( $2 \mu \mathrm{m}$ thick), and $\mathrm{n}+$ doped silicon (500 nm thick). Another PIN photodiode is underneath the nanowire PIN, in a back-to-back configuration. The external quantum efficiency (EQE) of the nanowire pixels is shown in Fig. 11(c), indicating spectrally tailored detector response and showing peak EQE values of 30\% when leaky hybrid (HE) waveguide modes are excited. Narrowband light sources across the visible spectrum are measured and reconstructed using a $\mathrm{L}_{1}$ regularization algorithm, ${ }^{69}$ with a spectral resolution of $\sim 5 \mathrm{~nm}$ achieved (Fig. $11(d))$.
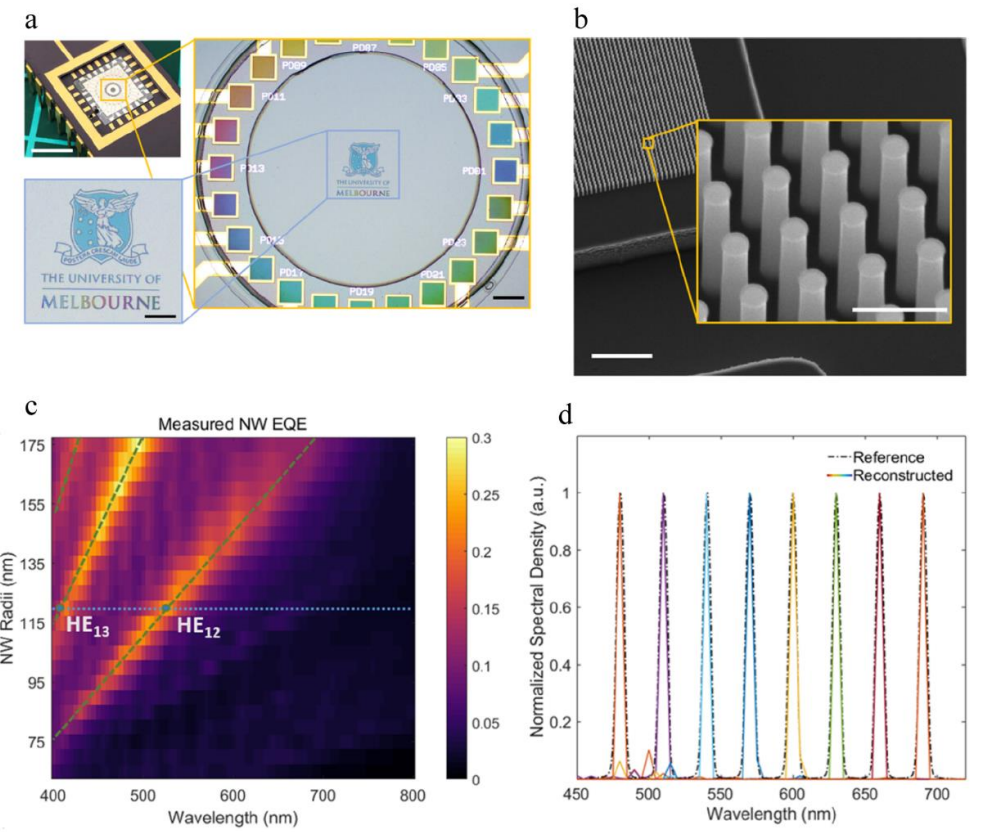

Fig. 11. Silicon nanowire array microspectrometer operating in visible spectral range. ${ }^{68}$ (a) Optical images of microspectrometer chip. Each pixel consists of an array of nanowires of particular radius, which in turn determines its structural coloration. Scale bars are (clockwise from top left): $5 \mathrm{~mm}, 200$ and $100 \mu \mathrm{m}$. (b) SEM image of pixel with nanowire array fabricated on PIN doped silicon substrate. Scale bars are 10 and $1 \mu \mathrm{m}$ (inset). (c) Measured EQE spectral of nanowire detector. (d) Reconstructed and reference (dashed lines) sample 
spectra. Reproduced with permission from J. J. Meng et al., Nano Lett. 20, 320 (2020). Copyright 2020 American Chemical Society.

Despite recent interest in microspectrometers, their operation wavelengths are mainly limited to the visible and near-infrared regions. Graphene MIR PDs offer opportunities for extending the operating wavelength of microspectrometers to the technologically important MIR region. The spectral response of graphene MIR PDs could be conveniently tailored using a variety of strategies reviewed in this paper, including coupling with colloidal quantum dots (CQDs) and topological semiconductors, integration with plasmonic nanostructures and exploiting graphene plasmons. In addition, due to the strong electrical tunability of the carrier density in graphene and the resultant change in permittivity, the graphene absorption spectrum could potentially be made to be electricallytunable by employing metal plasmon resonances, ${ }^{45}$ graphene plasmon resonances ${ }^{53}$ or hybrid resonances from both metal and graphene plasmons. ${ }^{70}$ This means that a series of distinct response functions could be obtained from a graphene PD by applying different gate voltages on the graphene layer, opening the possibility of a MIR microspectrometer consisting of just a single pixel.

In summary, we reviewed the recent progress of graphene-based MIR PDs, with an emphasis on state-of-the-art devices employing a variety of metamaterials and related concepts for responsivity enhancement. We conclude by providing potential directions for further improving the responsivity of graphene MIR PDs and discussing future applications these detectors may enable. With the continuing performance improvement of graphene MIR PDs, we envision that a wide range of emerging applications could be made possible in the future including next-generation biosensors, imaging sensors, and microspectrometers that are integrated with CMOS technology and implemented in wearable and portable devices.

\section{ACKNOWLEDGMENT}

This work was supported in part by the Australian Research Council (DP180104141 and CE200100010). 


\section{DATA AVAILABILITY}

Data sharing is not applicable to this article as no new data were created or analyzed in this study.

\section{REFERENCES}

$1 \quad$ Antoni Rogalski, Progress in Quantum Electronics 27 (2), 59 (2003); $\quad$ Antoni Rogalski, Progress in Quantum Electronics 36 (2), 342 (2012).

2 Sevgi Türker-Kaya and Christian W Huck, Molecules 22 (1), 168 (2017).

3 C Young, S-S Kim, Y Luzinova, M Weida, D Arnone, E Takeuchi, T Day, and B Mizaikoff, Sensors and Actuators B: Chemical 140 (1), 24 (2009); Daniel Rodrigo, Odeta Limaj, Davide Janner, Dordaneh Etezadi, F Javier García De Abajo, Valerio Pruneri, and Hatice Altug, Science 349 (6244), 165 (2015).

$4 \quad$ Timothy Day, Michael Pushkarsky, Dave Caffey, Kristen Cecchetti, Ron Arp, Alex Whitmore, Michael Henson, and Eric B Takeuchi, presented at the Technologies for Optical Countermeasures X; and High-Power Lasers 2013: Technology and Systems, 2013 (unpublished).

5 Frank K Tittel, Dirk Richter, and Alan Fried, in Solid-state mid-infrared laser sources (Springer, 2003), pp. 458.

6 Bujin Guo, Y Wang, C Peng, HL Zhang, GP Luo, HQ Le, Claire Gmachl, DL Sivco, ML Peabody, and Alfred Y Cho, Optics express 12 (1), 208 (2004); Manijeh Razeghi and Binh-Minh Nguyen, Reports on Progress in Physics 77 (8), 082401 (2014); Xin Tang, Matthew M Ackerman, Menglu Chen, and Philippe Guyot-Sionnest, Nature Photonics 13 (4), 277 (2019).

7 Callum J Docherty, Cheng-Te Lin, Hannah J Joyce, Robin J Nicholas, Laura M Herz, Lain-Jong Li, and Michael B Johnston, Nature communications 3 (1), 1 (2012); Shengxue Yang, Chengbao Jiang, and Su-huai Wei, Applied Physics Reviews 4 (2), 021304 (2017).

8 Genevieve Gariepy, Francesco Tonolini, Robert Henderson, Jonathan Leach, and Daniele Faccio, Nature Photonics 10 (1), 23 (2016); Lu-Qi Tao, Kun-Ning Zhang, He Tian, Ying Liu, Dan-Yang Wang, Yuan-Quan Chen, Yi Yang, and Tian-Ling Ren, ACS nano 11 (9), 8790 (2017).

9 Heinrich Figgemeier, Christopher Ames, Johannes Beetz, Rainer Breiter, Detlef Eich, Stefan Hanna, Karl-Martin Mahlein, Timo Schallenberg, Alexander Sieck, and Jan Wenisch, presented at the Infrared Technology and Applications XLIV, 2018 (unpublished); C Cervera, N Baier, O Gravrand, L Mollard, C Lobre, G Destefanis, JP Zanatta, O Boulade, and V Moreau, presented at the Infrared Technology and Applications XLI, 2015 (unpublished). E Neil Lewis and Ira W Levin, Applied spectroscopy 49 (5), 672 (1995).

11 Binbin Weng, Jijun Qiu, Lihua Zhao, Zijian Yuan, Caleb Chang, and Zhisheng Shi, presented at the Quantum Sensing and Nanophotonic Devices XI, 2014 (unpublished).

12 FHL Koppens, T Mueller, Ph Avouris, AC Ferrari, MS Vitiello, and M Polini, Nature nanotechnology 9 (10), 780 (2014); $\quad$ Rahul Raveendran Nair, Peter Blake, Alexander N Grigorenko, Konstantin S Novoselov, Tim J Booth, Tobias Stauber, Nuno MR Peres, and Andre K Geim, Science 320 (5881), 1308 (2008); Kin Fai Mak, Long Ju, Feng Wang, and Tony F Heinz, Solid State Communications 152 (15), 1341 (2012); $\quad$ Sichao Du, Wei Lu, Ayaz Ali, Pei Zhao, Khurram Shehzad, Hongwei Guo, Lingling Ma, Xuemei Liu, Xiaodong Pi, and Peng Wang, Advanced Materials 29 (22), 1700463 (2017). Adolfo De Sanctis, Gareth F Jones, Dominique J Wehenkel, Francisco Bezares, Frank HL Koppens, Monica F Craciun, and Saverio Russo, Science advances 3 (5), e1602617 (2017); Marcus Freitag, Tony Low, Wenjuan Zhu, Hugen Yan, Fengnian Xia, and Phaedon Avouris, Nature communications 4, 1951 (2013); Tao Deng, Zhaohao Zhang, Yaxuan Liu, Yingxin Wang, Fang Su, Shasha Li, Yang Zhang, Hao Li, Houjin Chen, and Ziran Zhao, Nano letters 19 (3), 1494 (2019); Sebastián Castilla, Bernat Terrés, Marta Autore, Leonardo Viti, Jian Li, Alexey Y Nikitin, loannis Vangelidis, Kenji Watanabe, Takashi Taniguchi, and Elefterios Lidorikis, Nano letters 19 (5), 2765 (2019).

14 Fengnian Xia, Thomas Mueller, Yu-ming Lin, Alberto Valdes-Garcia, and Phaedon Avouris, Nature nanotechnology 4 (12), 839 (2009). 
Thomas Mueller, Fengnian Xia, and Phaedon Avouris, Nature photonics 4 (5), 297 (2010). J Piotrowski, Opto-Electron. Rev 12 (1), 111 (2004).

Deji Akinwande, Cedric Huyghebaert, Ching-Hua Wang, Martha I. Serna, Stijn Goossens, Lain-Jong Li, H. S. Philip Wong, and Frank H. L. Koppens, Nature 573 (7775), 507 (2019); $\quad$ F. Bonaccorso, Z. Sun, T. Hasan, and A. C. Ferrari, Nature Photonics 4 (9), 611 (2010); Marco Romagnoli, Vito Sorianello, Michele Midrio, Frank H. L. Koppens, Cedric Huyghebaert, Daniel Neumaier, Paola Galli, Wolfgang Templ, Antonio D'Errico, and Andrea C. Ferrari, Nature Reviews Materials 3 (10), 392 (2018); K. S. Novoselov, V. I. Fal'ko, L. Colombo, P. R. Gellert, M. G. Schwab, and K. Kim, Nature 490 (7419), 192 (2012).

A. H. Castro Neto, F. Guinea, N. M. R. Peres, K. S. Novoselov, and A. K. Geim, Reviews of Modern Physics 81 (1), 109 (2009); $\quad$ A. K. Geim and K. S. Novoselov, Nature Materials 6 (3), 183 (2007). Andre K Geim and Konstantin S Novoselov, in Nanoscience and technology: a collection of reviews from nature journals (World Scientific, 2010), pp. 11.

Nathaniel M Gabor, Justin CW Song, Qiong Ma, Nityan L Nair, Thiti Taychatanapat, Kenji Watanabe, Takashi Taniguchi, Leonid S Levitov, and Pablo Jarillo-Herrero, Science 334 (6056), 648 (2011).

Michela Badioli, Achim Woessner, Klaas-Jan Tielrooij, Sebastien Nanot, Gabriele Navickaite, Tobias Stauber, FJ García de Abajo, and Frank HL Koppens, Nano letters 14 (11), 6374 (2014).

InfraRed Associates, Inc., LN2 Cooled HgCdTe Detectors. Retrieved from http://irassociates.com/index.php?page=ln2-cooled.

InfraRed Associates, Inc., InSb Detectors. Retrieved from http://irassociates.com/index.php?page=insb.

Thorlabs, Inc., FDPSE2X2 - PbSe Photoconductor. Retrieved from https://www.thorlabs.com/thorproduct.cfm?partnumber=FDPSE2X2\&pn=FDPSE2X2\#6197

Justin CW Song, Mark S Rudner, Charles M Marcus, and Leonid S Levitov, Nano letters 11 (11), 4688 (2011); Xiaodong Xu, Nathaniel M Gabor, Jonathan S Alden, Arend M van der Zande, and Paul L McEuen, Nano letters 10 (2), 562 (2010); Dong Sun, Grant Aivazian, Aaron M Jones, Jason S Ross, Wang Yao, David Cobden, and Xiaodong Xu, Nature nanotechnology 7 (2), 114 (2012); Ermin Malic, Torben Winzer, Florian Wendler, and Andreas Knorr, physica status solidi (b) 253 (12), 2303 (2016); K. I. Bolotin, K. J. Sikes, Z. Jiang, M. Klima, G. Fudenberg, J. Hone, P. Kim, and H. L. Stormer, Solid State Communications 146 (9), 351 (2008)

Valeri N Kotov, Bruno Uchoa, Vitor M Pereira, F Guinea, and AH Castro Neto, Reviews of Modern Physics 84 (3), 1067 (2012).

Klaas-Jan Tielrooij, JCW Song, Soren A Jensen, A Centeno, A Pesquera, A Zurutuza Elorza, Mischa Bonn, LS Levitov, and FHL Koppens, Nature Physics 9 (4), 248 (2013); Justin CW Song, Klaas J Tielrooij, Frank HL Koppens, and Leonid S Levitov, Physical Review B 87 (15), 155429 (2013); Isabella Gierz, Jesse C Petersen, Matteo Mitrano, Cephise Cacho, IC Edmond Turcu, Emma Springate, Alexander Stöhr, Axel Köhler, Ulrich Starke, and Andrea Cavalleri, Nature materials 12 (12), 1119 (2013); Jens Christian Johannsen, Søren Ulstrup, Federico Cilento, Alberto Crepaldi, Michele Zacchigna, Cephise Cacho, IC Edmond Turcu, Emma Springate, Felix Fromm, and Christian Raidel, Physical Review Letters 111 (2), 027403 (2013).

R Bistritzer and Allan H MacDonald, Physical Review Letters 102 (20), 206410 (2009); Wang-

Kong Tse and S Das Sarma, Physical Review B 79 (23), 235406 (2009).

Cheng Peng, Sebastien Nanot, Ren-Jye Shiue, Gabriele Grosso, Yafang Yang, Marek Hempel, Pablo Jarillo-Herrero, Jing Kong, Frank HL Koppens, and Dmitri K Efetov, New Journal of Physics 20 (8), 083050 (2018).

Qiushi Guo, Renwen Yu, Cheng Li, Shaofan Yuan, Bingchen Deng, F Javier García de Abajo, and Fengnian Xia, Nature materials 17 (11), 986 (2018).

Marcus Freitag, Tony Low, Wenjuan Zhu, Hugen Yan, Fengnian Xia, and Phaedon Avouris, Nature communications 4 (1), 1 (2013).

Eva C Peters, Eduardo JH Lee, Marko Burghard, and Klaus Kern, Applied Physics Letters 97 (19), 193102 (2010). 
Gayathri Rao, Marcus Freitag, Hsin-Ying Chiu, Ravi S Sundaram, and Phaedon Avouris, ACS nano 5 (7), 5848 (2011); $\quad$ T Mueller, F Xia, M Freitag, J Tsang, and Ph Avouris, Physical Review B 79 (24), 245430 (2009).

Damon B Farmer, Roksana Golizadeh-Mojarad, Vasili Perebeinos, Yu-Ming Lin, George S Tulevski, James C Tsang, and Phaedon Avouris, Nano letters 9 (1), 388 (2009).

Max C Lemme, Frank HL Koppens, Abram L Falk, Mark S Rudner, Hongkun Park, Leonid S Levitov, and Charles M Marcus, Nano letters 11 (10), 4134 (2011).

Marcus Freitag, Tony Low, Fengnian Xia, and Phaedon Avouris, Nature Photonics 7 (1), 53 (2013). Andrea Tomadin, Daniele Brida, Giulio Cerullo, Andrea C. Ferrari, and Marco Polini, Physical Review B 88 (3), 035430 (2013); $\quad$ Ermin Malic, Torben Winzer, Evgeny Bobkin, and Andreas Knorr, Physical Review B 84 (20), 205406 (2011); Raseong Kim, Vasili Perebeinos, and Phaedon Avouris, Physical Review B 84 (7), 075449 (2011).

D. Brida, A. Tomadin, C. Manzoni, Y. J. Kim, A. Lombardo, S. Milana, R. R. Nair, K. S. Novoselov, A. C. Ferrari, G. Cerullo, and M. Polini, Nature Communications 4 (1), 1987 (2013); Torben Winzer, Andreas Knorr, and Ermin Malic, Nano Letters 10 (12), 4839 (2010).

Yu Yao, Raji Shankar, Patrick Rauter, Yi Song, Jing Kong, Marko Loncar, and Federico Capasso, Nano letters 14 (7), 3749 (2014)

U Sassi, Romain Parret, S Nanot, M Bruna, S Borini, Domenico De Fazio, Z Zhao, E Lidorikis, FHL Koppens, and Andrea Carlo Ferrari, Nature communications 8 (1), 1 (2017).

Kavitha K Gopalan, Davide Janner, Sebastien Nanot, Romain Parret, Mark B Lundeberg, Frank HL Koppens, and Valerio Pruneri, Advanced Optical Materials 5 (4), 1600723 (2017).

Yongzhe Zhang, Tao Liu, Bo Meng, Xiaohui Li, Guozhen Liang, Xiaonan Hu, and Qi Jie Wang, Nature communications 4, 1811 (2013).

Xuechao Yu, Zhaogang Dong, Yanping Liu, Tao Liu, Jin Tao, Yongquan Zeng, Joel KW Yang, and Qi Jie Wang, Nanoscale 8 (1), 327 (2016); Xuechao Yu, Zhaogang Dong, Joel KW Yang, and Qi Jie Wang, Optica 3 (9), 979 (2016).

Xuechao Yu, Yangyang Li, Xiaonan Hu, Daliang Zhang, Ye Tao, Zhixiong Liu, Yongmin He, Md Azimul Haque, Zheng Liu, and Tom Wu, Nature communications 9 (1), 4299 (2018).

Jaeseok Kim, Sungjoon Park, Houk Jang, Nikesh Koirala, Jae-Bok Lee, Un Jeong Kim, Hong-Seok Lee, Young-Geun Roh, Hyangsook Lee, Sangwan Sim, Soonyoung Cha, Chihun In, Jun Park, Jekwan Lee, Minji Noh, Jisoo Moon, Maryam Salehi, Jiho Sung, Sang-Soo Chee, Moon-Ho Ham, Moon-Ho Jo, Seongshik Oh, Jong-Hyun Ahn, Sung Woo Hwang, Dohun Kim, and Hyunyong Choi, ACS Photonics 4 (3), 482 (2017).

Chang-Hua Liu, You-Chia Chang, Theodore B Norris, and Zhaohui Zhong, Nature nanotechnology 9 (4), 273 (2014).

Ming Ye, Yang Gao, Jasper J. Cadusch, Sivacarendran Balendhran, and Kenneth B. Crozier, Advanced Optical Materials 9 (6), 2001854 (2021).

Zhenyi Ni, Lingling Ma, Sichao Du, Yang Xu, Meng Yuan, Hehai Fang, Zhen Wang, Mingsheng Xu, Dongsheng Li, Jianyi Yang, Weida Hu, Xiaodong Pi, and Deren Yang, ACS Nano 11 (10), 9854 (2017). Semih Cakmakyapan, Ping Keng Lu, Aryan Navabi, and Mona Jarrahi, Light: Science \& Applications 7 (1), 20 (2018).

Jingxuan Wei, Ying Li, Lin Wang, Wugang Liao, Bowei Dong, Cheng Xu, Chunxiang Zhu, Kah-Wee Ang, Cheng-Wei Qiu, and Chengkuo Lee, Nature Communications 11 (1), 6404 (2020).

Nima Sefidmooye Azar, Vivek Raj Shrestha, and Kenneth B Crozier, Applied Physics Letters 114 (9), 091108 (2019).

Xiaomu Wang, Zhenzhou Cheng, Ke Xu, Hon Ki Tsang, and Jian-Bin Xu, Nature Photonics 7 (11), 888 (2013).

Alireza Safaei, Sayan Chandra, Muhammad Waqas Shabbir, Michael N Leuenberger, and Debashis Chanda, Nature communications 10 (1), 1 (2019).

Achim Woessner, Romain Parret, Diana Davydovskaya, Yuanda Gao, Jhih-Sheng Wu, Mark B. Lundeberg, Sébastien Nanot, Pablo Alonso-González, Kenji Watanabe, Takashi Taniguchi, Rainer 
Hillenbrand, Michael M. Fogler, James Hone, and Frank H. L. Koppens, npj 2D Materials and Applications 1 (1), 25 (2017).

Sebastián Castilla, Ioannis Vangelidis, Varun-Varma Pusapati, Jordan Goldstein, Marta Autore, Tetiana Slipchenko, Khannan Rajendran, Seyoon Kim, Kenji Watanabe, Takashi Taniguchi, Luis Martín-Moreno, Dirk Englund, Klaas-Jan Tielrooij, Rainer Hillenbrand, Elefterios Lidorikis, and Frank H. L. Koppens, Nature Communications 11 (1), 4872 (2020). Bingchen Deng, Chao Ma, Qiyue Wang, Shaofan Yuan, Kenji Watanabe, Takashi Taniguchi, Fan Zhang, and Fengnian Xia, Nature Photonics 14 (9), 549 (2020). Randy J Ellingson, Matthew C Beard, Justin C Johnson, Pingrong Yu, Olga I Micic, Arthur J Nozik, Andrew Shabaev, and Alexander L Efros, Nano letters 5 (5), 865 (2005). Hehai Fang and Weida Hu, Advanced science 4 (12), 1700323 (2017). (2018); Zheyu Fang, Yumin Wang, Andrea E Schlather, Zheng Liu, Pulickel M Ajayan, F Javier García de Abajo, Peter Nordlander, Xing Zhu, and Naomi J Halas, Nano letters 14 (1), 299 (2014).

Rahul Raveendran Nair, Peter Blake, Alexander N Grigorenko, Konstantin S Novoselov, Tim J Booth, Tobias Stauber, Nuno MR Peres, and Andre K Geim, Science 320 (5881), 1308 (2008); Kin Fai Mak, Matthew Y Sfeir, Yang Wu, Chun Hung Lui, James A Misewich, and Tony F Heinz, Physical review letters 101 (19), 196405 (2008).

K. J. Tielrooij, L. Piatkowski, M. Massicotte, A. Woessner, Q. Ma, Y. Lee, K. S. Myhro, C. N. Lau, P. Jarillo-Herrero, N. F. van Hulst, and F. H. L. Koppens, Nature Nanotechnology 10 (5), 437 (2015); Klaas-Jan Tielrooij, Niels C. H. Hesp, Alessandro Principi, Mark B. Lundeberg, Eva A. A. Pogna, Luca Banszerus, Zoltán Mics, Mathieu Massicotte, Peter Schmidt, Diana Davydovskaya, David G. Purdie, Ilya Goykhman, Giancarlo Soavi, Antonio Lombardo, Kenji Watanabe, Takashi Taniguchi, Mischa Bonn, Dmitry Turchinovich, Christoph Stampfer, Andrea C. Ferrari, Giulio Cerullo, Marco Polini, and Frank H. L. Koppens, Nature Nanotechnology 13 (1), 41 (2018); Justin C. W. Song, Mark S. Rudner, Charles M. Marcus, and Leonid S. Levitov, Nano Letters 11 (11), 4688 (2011). Allen L. Hsu, Patrick K. Herring, Nathaniel M. Gabor, Sungjae Ha, Yong Cheol Shin, Yi Song, Matthew Chin, Madan Dubey, Anantha P. Chandrakasan, Jing Kong, Pablo Jarillo-Herrero, and Tomás Palacios, Nano Letters 15 (11), 7211 (2015); Patrick K. Herring, Allen L. Hsu, Nathaniel M. Gabor, Yong Cheol Shin, Jing Kong, Tomás Palacios, and Pablo Jarillo-Herrero, Nano Letters 14 (2), 901 (2014). Rafi Bistritzer and Allan H. MacDonald, Proceedings of the National Academy of Sciences 108 (30), 12233 (2011); Y. Cao, J. Y Luo, V. Fatemi, S. Fang, J. D Sanchez-Yamagishi, K. Watanabe, T. Taniguchi, E. Kaxiras, and P. Jarillo-Herrero, Physical Review Letters 117 (11), 116804 (2016); Kyounghwan Kim, Ashley DaSilva, Shengqiang Huang, Babak Fallahazad, Stefano Larentis, Takashi Taniguchi, Kenji Watanabe, Brian J. LeRoy, Allan H. MacDonald, and Emanuel Tutuc, Proceedings of the National Academy of Sciences 114 (13), 3364 (2017); Yuan Cao, Valla Fatemi, Shiang Fang, Kenji Watanabe, Takashi Taniguchi, Efthimios Kaxiras, and Pablo Jarillo-Herrero, Nature 556 (7699), 43 (2018); Yuan Cao, Valla Fatemi, Ahmet Demir, Shiang Fang, Spencer L. Tomarken, Jason Y. Luo, Javier D. Sanchez-Yamagishi, Kenji Watanabe, Takashi Taniguchi, Efthimios Kaxiras, Ray C. Ashoori, and Pablo Jarillo-Herrero, Nature 556 (7699), 80 (2018); Matthew Yankowitz, Shaowen Chen, Hryhoriy Polshyn, Yuxuan Zhang, K. Watanabe, T. Taniguchi, David Graf, Andrea F. Young, and Cory R. Dean, Science 363 (6431), 1059 (2019); J. M. B. Lopes dos Santos, N. M. R. Peres, and A. H. Castro Neto, Physical Review Letters 99 (25), 256802 (2007); E. J. Mele, Physical Review B 81 (16), 161405 (2010).

Kallol Roy, Medini Padmanabhan, Srijit Goswami, T. Phanindra Sai, Gopalakrishnan Ramalingam, Srinivasan Raghavan, and Arindam Ghosh, Nature Nanotechnology 8 (11), 826 (2013); Wenjing Zhang, Chih-Piao Chuu, Jing-Kai Huang, Chang-Hsiao Chen, Meng-Lin Tsai, Yung-Huang Chang, Chi-Te Liang, Yu-Ze Chen, Yu-Lun Chueh, Jr-Hau He, Mei-Yin Chou, and Lain-Jong Li, Scientific Reports 4 (1), 3826 (2014). Jake D. Mehew, Selim Unal, Elias Torres Alonso, Gareth F. Jones, Saad Fadhil Ramadhan, Monica F. Craciun, and Saverio Russo, Advanced Materials 29 (23), 1700222 (2017). 
Xiaolong Chen, Xiaobo Lu, Bingchen Deng, Ofer Sinai, Yuchuan Shao, Cheng Li, Shaofan Yuan, Vy Tran, Kenji Watanabe, Takashi Taniguchi, Doron Naveh, Li Yang, and Fengnian Xia, Nature Communications 8 (1), 1672 (2017); $\quad$ Matin Amani, Emma Regan, James Bullock, Geun Ho Ahn, and Ali Javey, ACS Nano 11 (11), 11724 (2017); Bingchen Deng, Vy Tran, Yujun Xie, Hao Jiang, Cheng Li, Qiushi Guo, Xiaomu Wang, He Tian, Steven J. Koester, Han Wang, Judy J. Cha, Qiangfei Xia, Li Yang, and Fengnian Xia, Nature Communications 8 (1), 14474 (2017); Mingsheng Long, Anyuan Gao, Peng Wang, Hui Xia, Claudia Ott, Chen Pan, Yajun Fu, Erfu Liu, Xiaoshuang Chen, Wei Lu, Tom Nilges, Jianbin Xu, Xiaomu Wang, Weida Hu, and Feng Miao, Science Advances 3 (6), e1700589 (2017); James Bullock, Matin Amani, Joy Cho, Yu-Ze Chen, Geun Ho Ahn, Valerio Adinolfi, Vivek Raj Shrestha, Yang Gao, Kenneth B. Crozier, Yu-Lun Chueh, and Ali Javey, Nature Photonics 12 (10), 601 (2018).

Zongyin Yang, Tom Albrow-Owen, Hanxiao Cui, Jack Alexander-Webber, Fuxing Gu, Xiaomu Wang, Tien-Chun Wu, Minghua Zhuge, Calum Williams, Pan Wang, Anatoly V. Zayats, Weiwei Cai, Lun Dai, Stephan Hofmann, Mauro Overend, Limin Tong, Qing Yang, Zhipei Sun, and Tawfique Hasan, Science 365 (6457), 1017 (2019); Jasper J. Cadusch, Jiajun Meng, Benjamin Craig, and Kenneth B. Crozier, Optica 6 (9), 1171 (2019). 

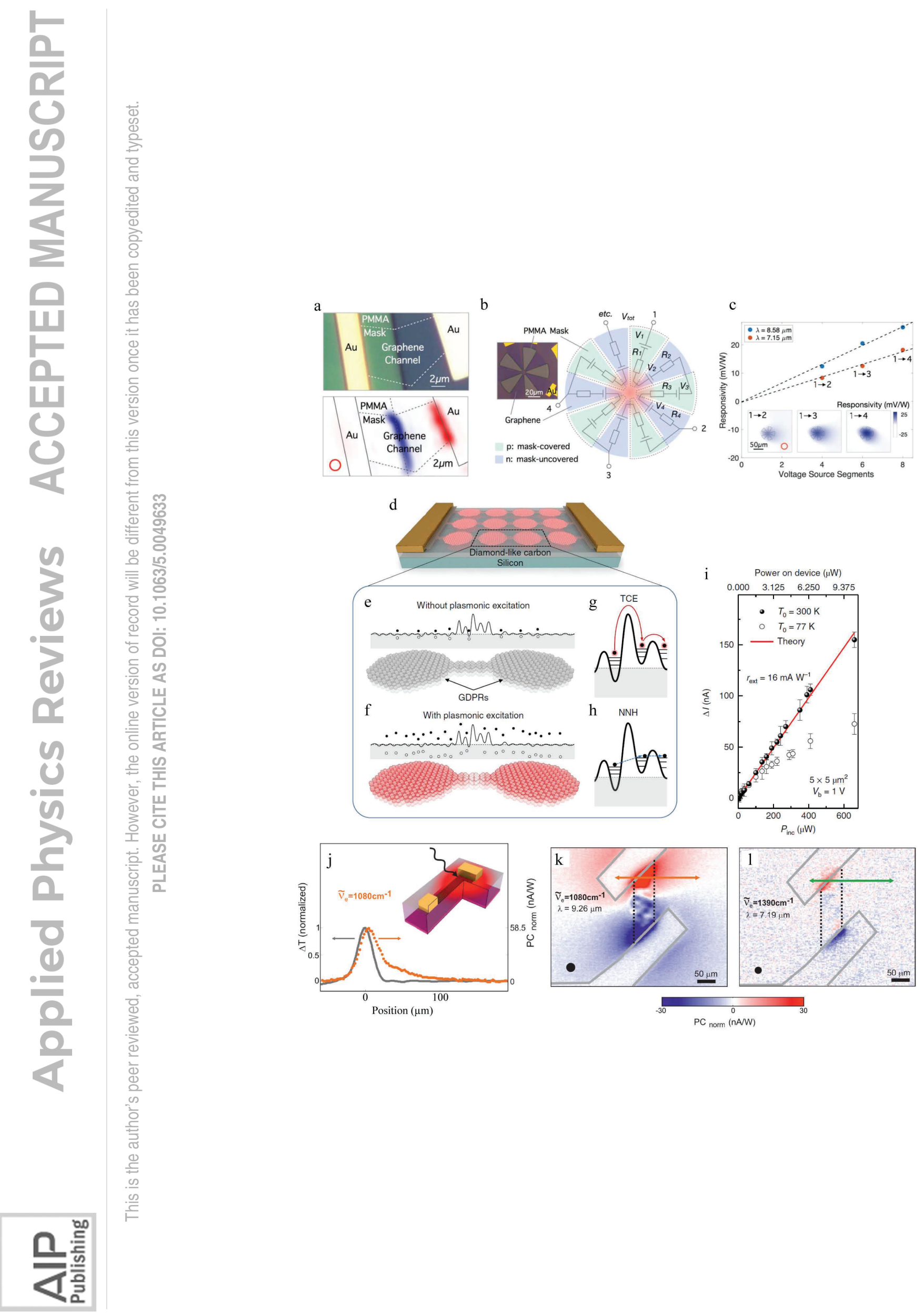
a

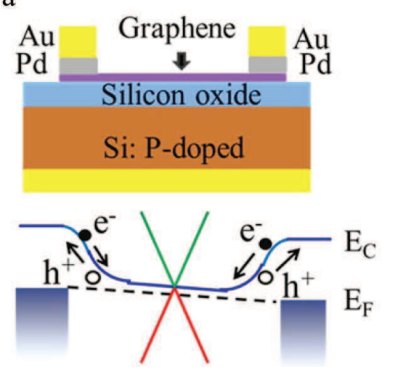

b

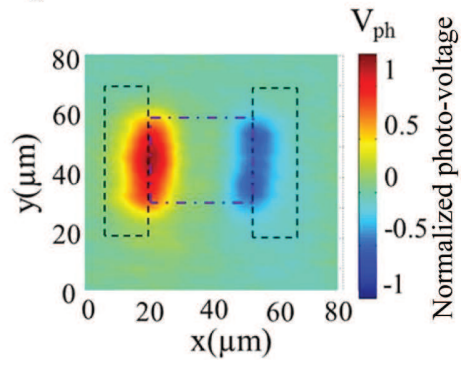



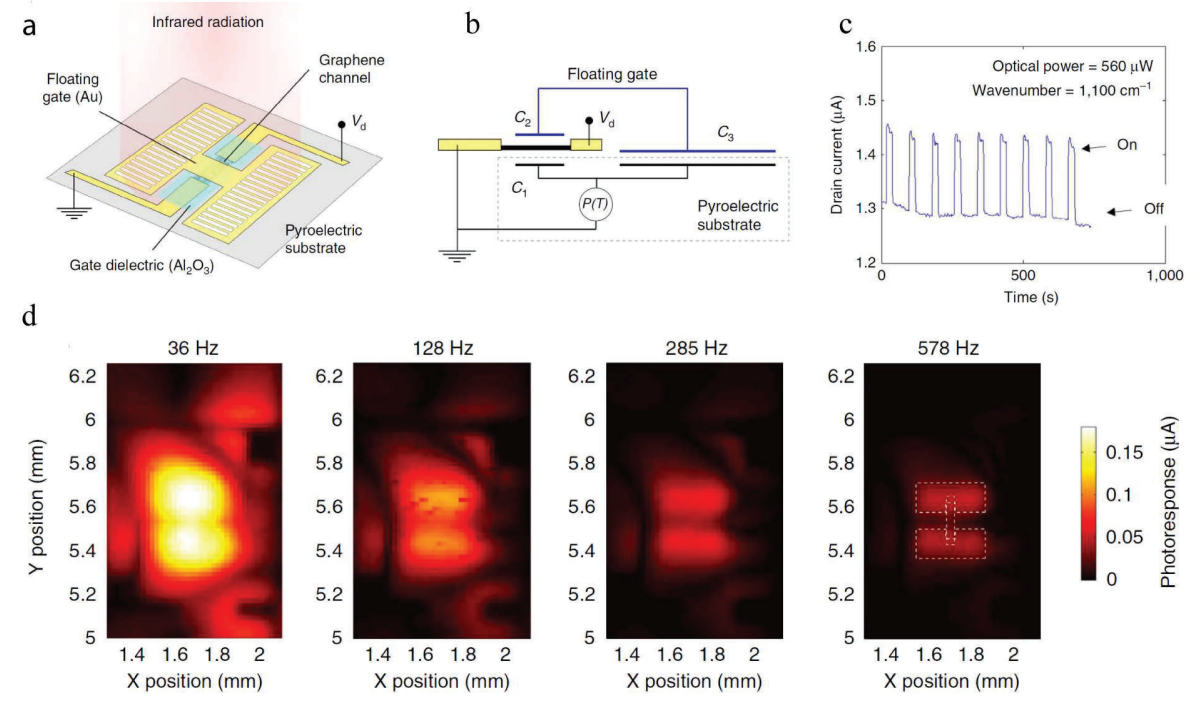

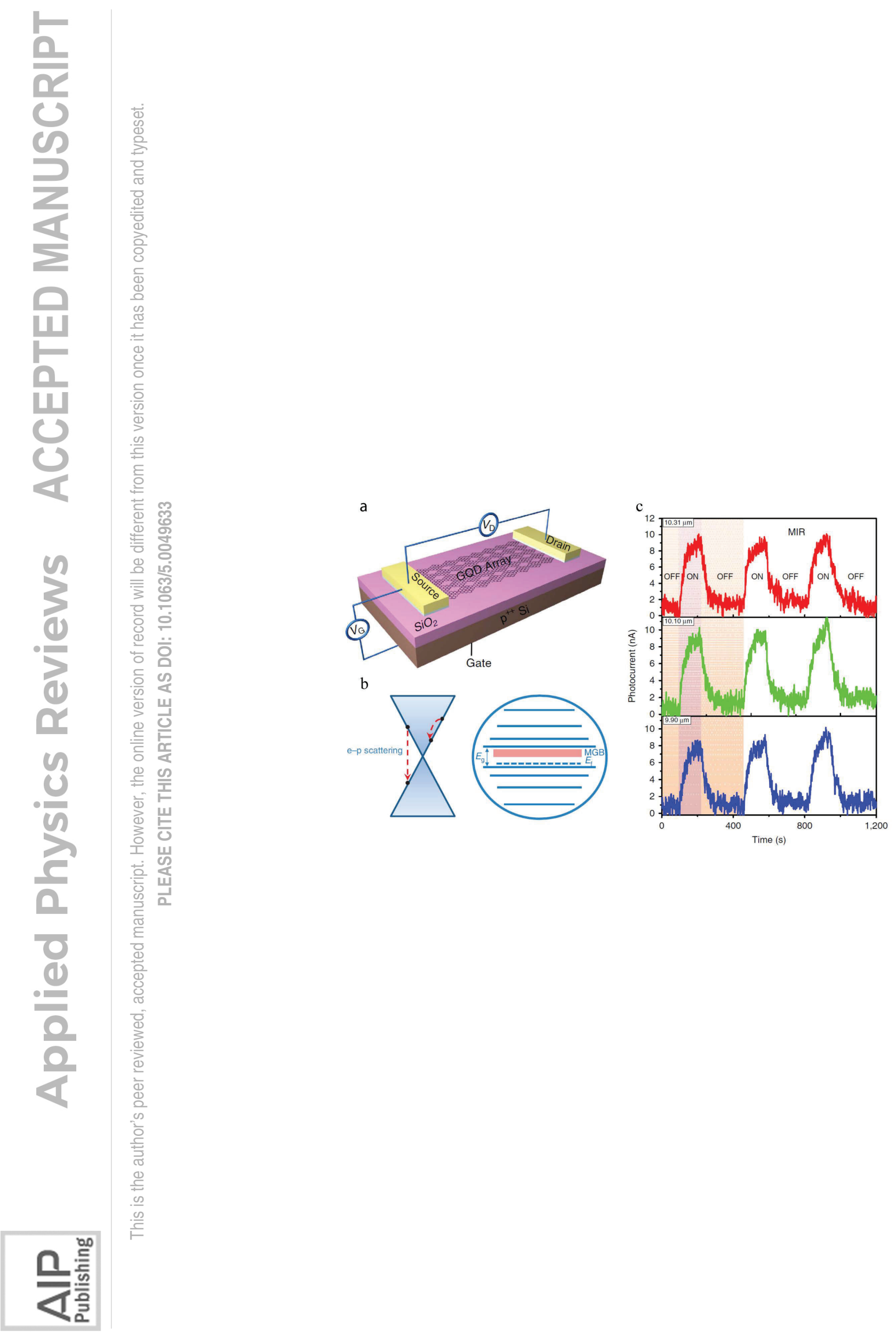

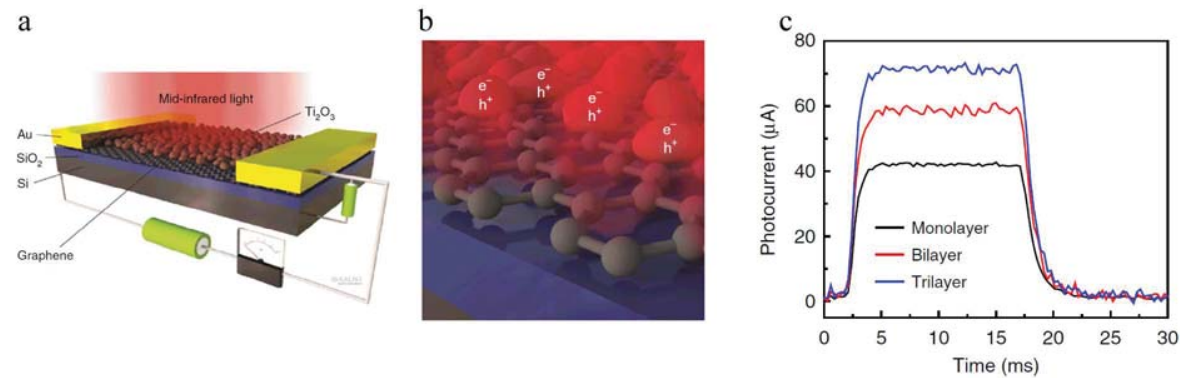

d

e
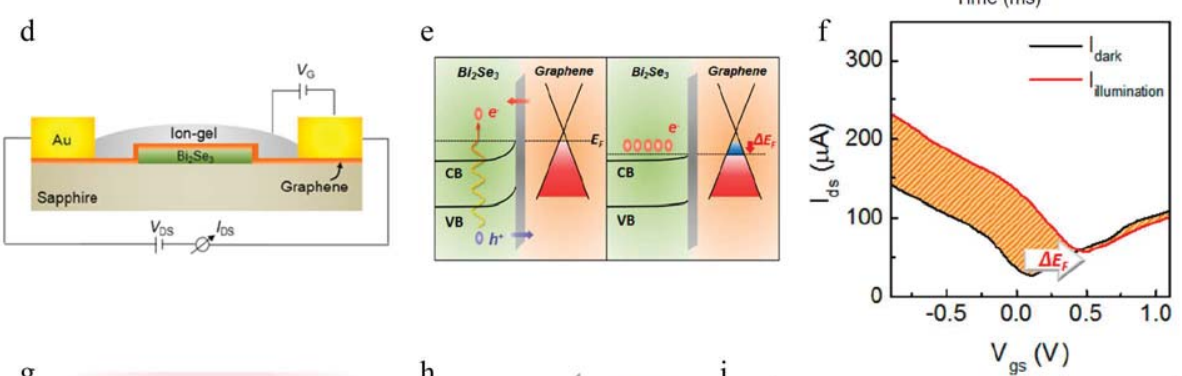

g

h
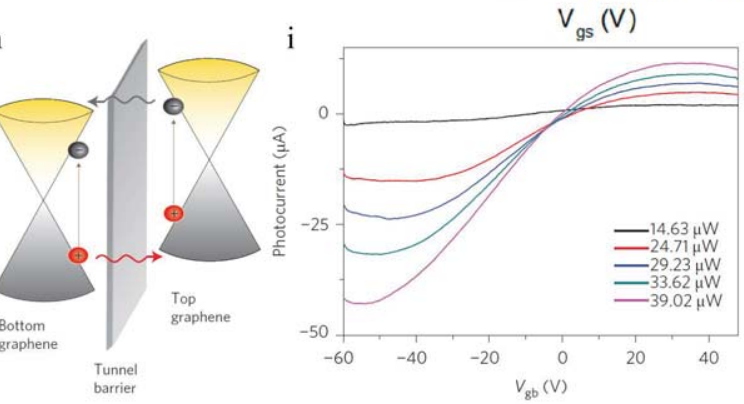

$\frac{1}{0}$ 

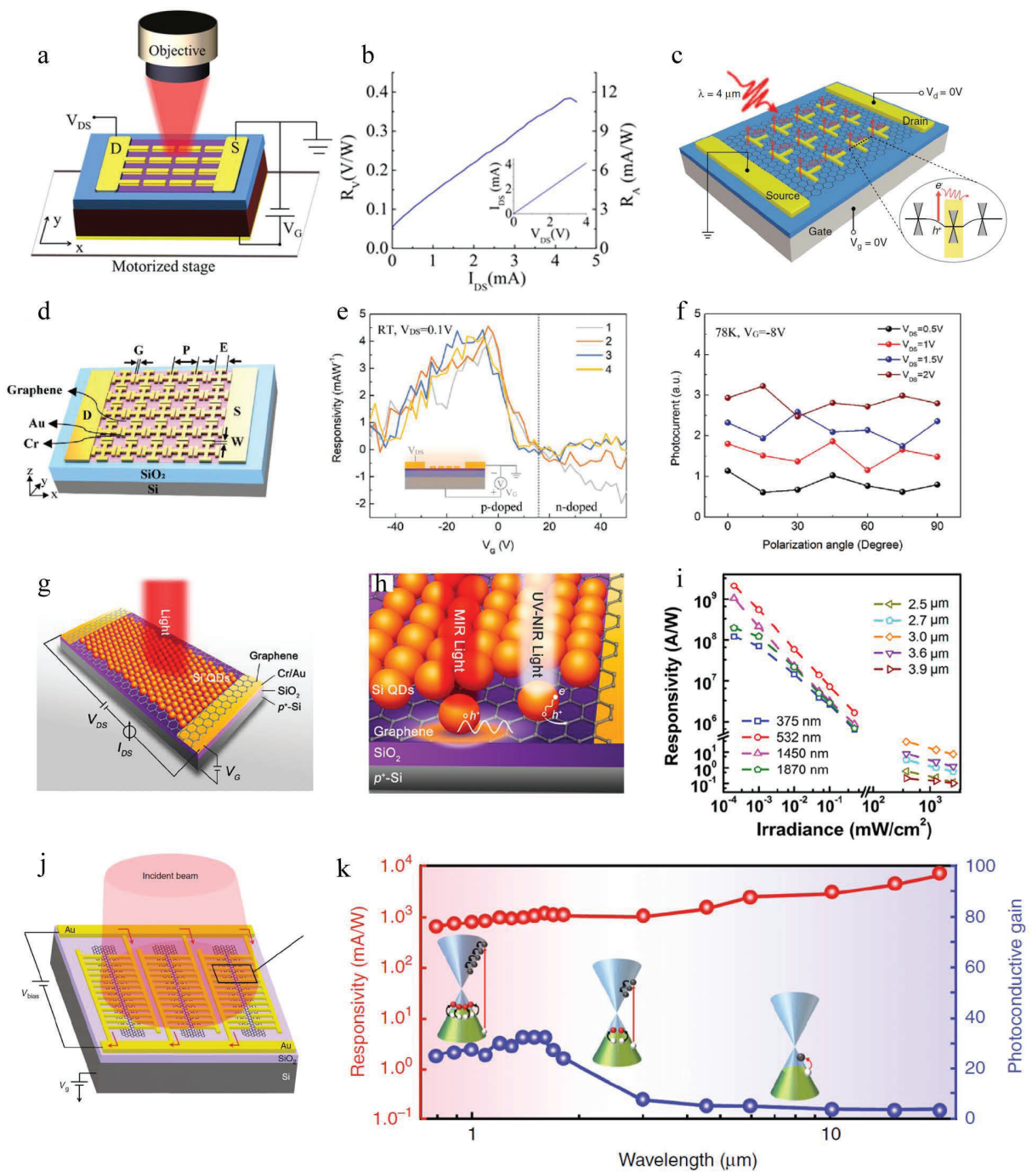

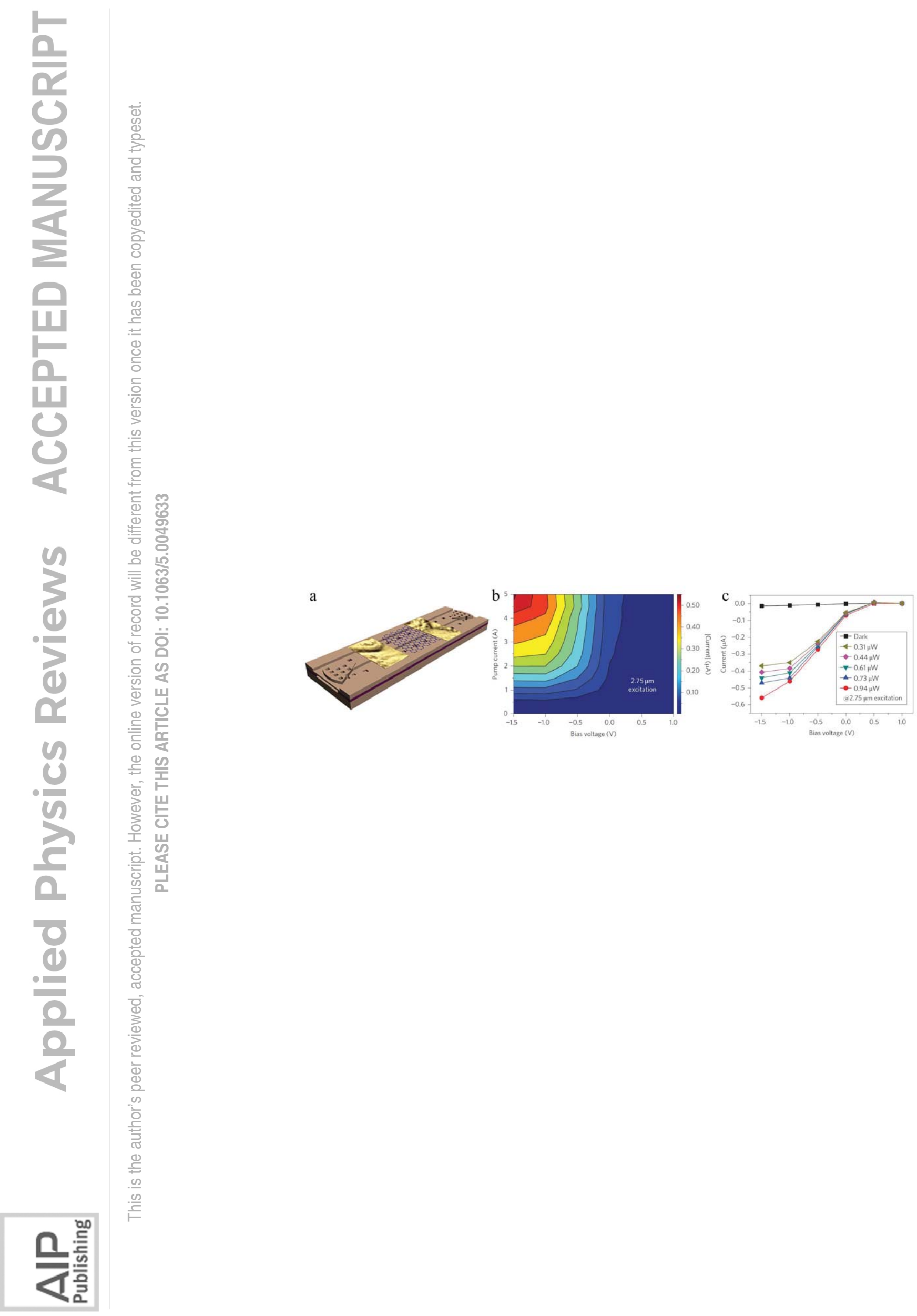

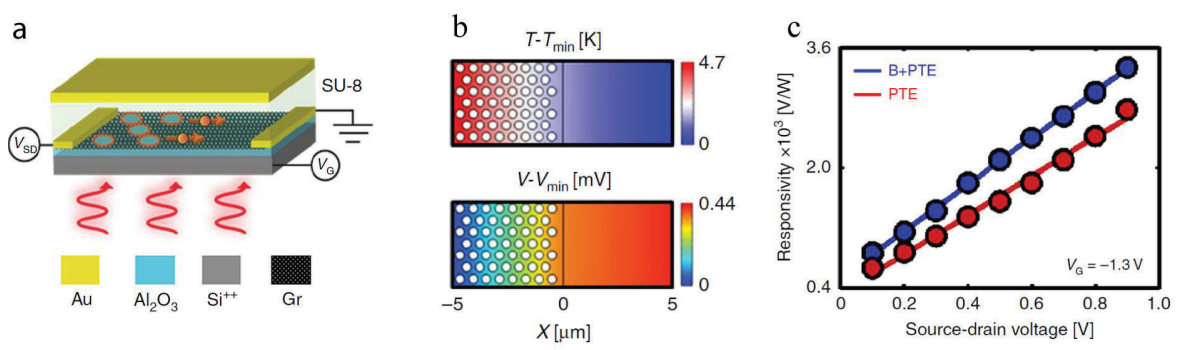

0
0
0
0
0
0
0
0

5

(1)

O

(

4

败喜 

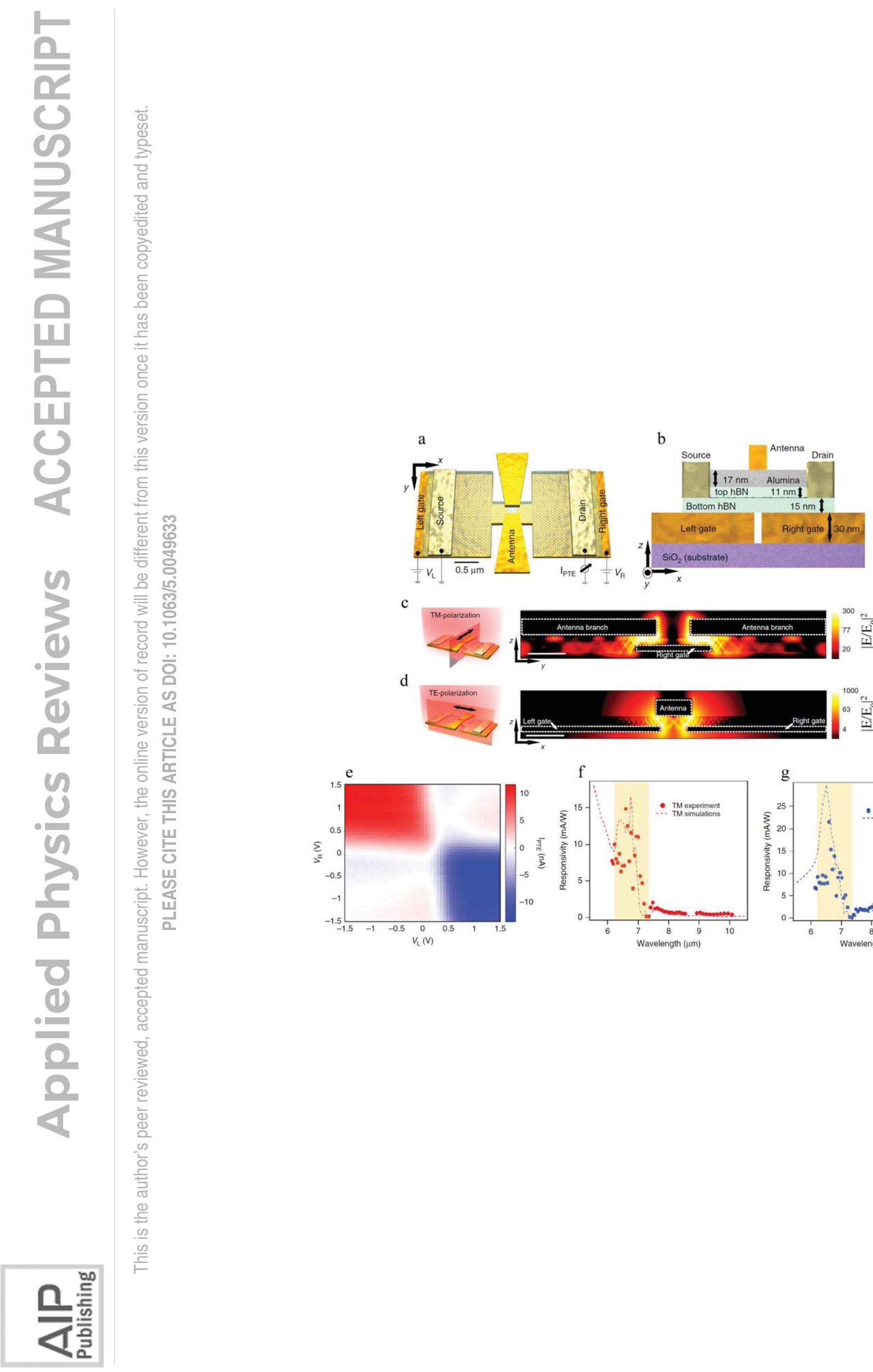

c

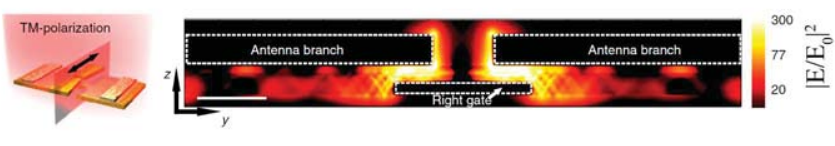

d
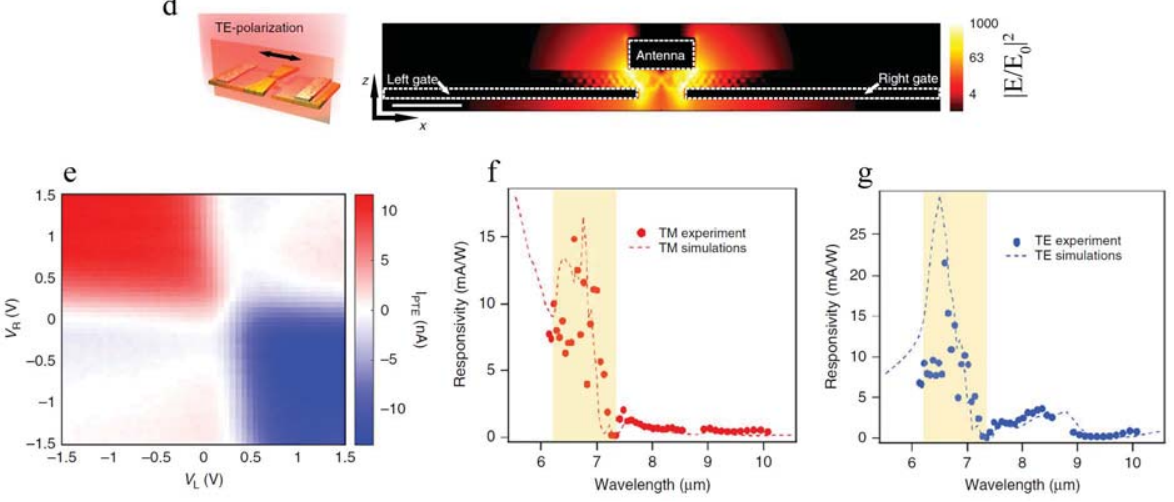

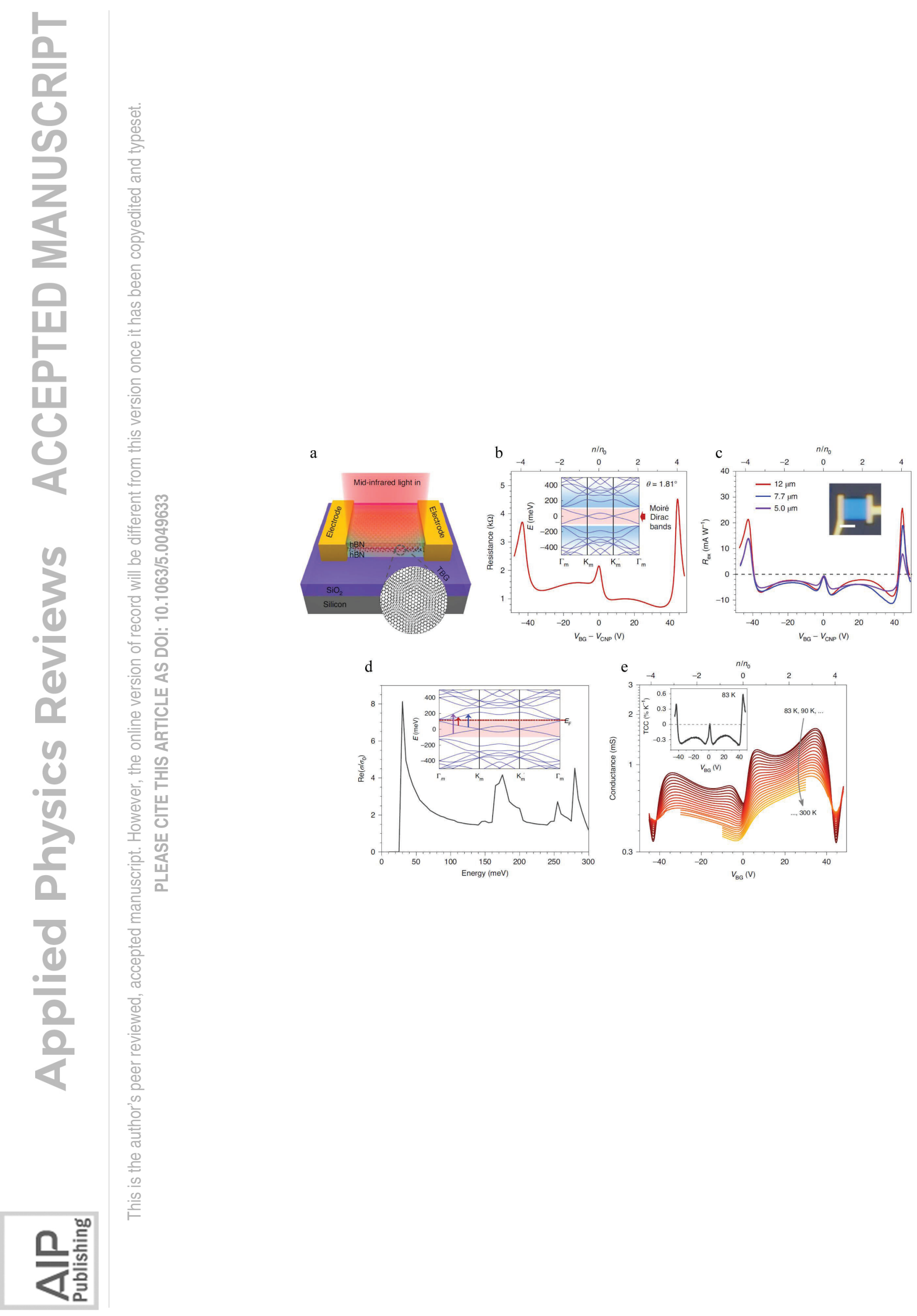

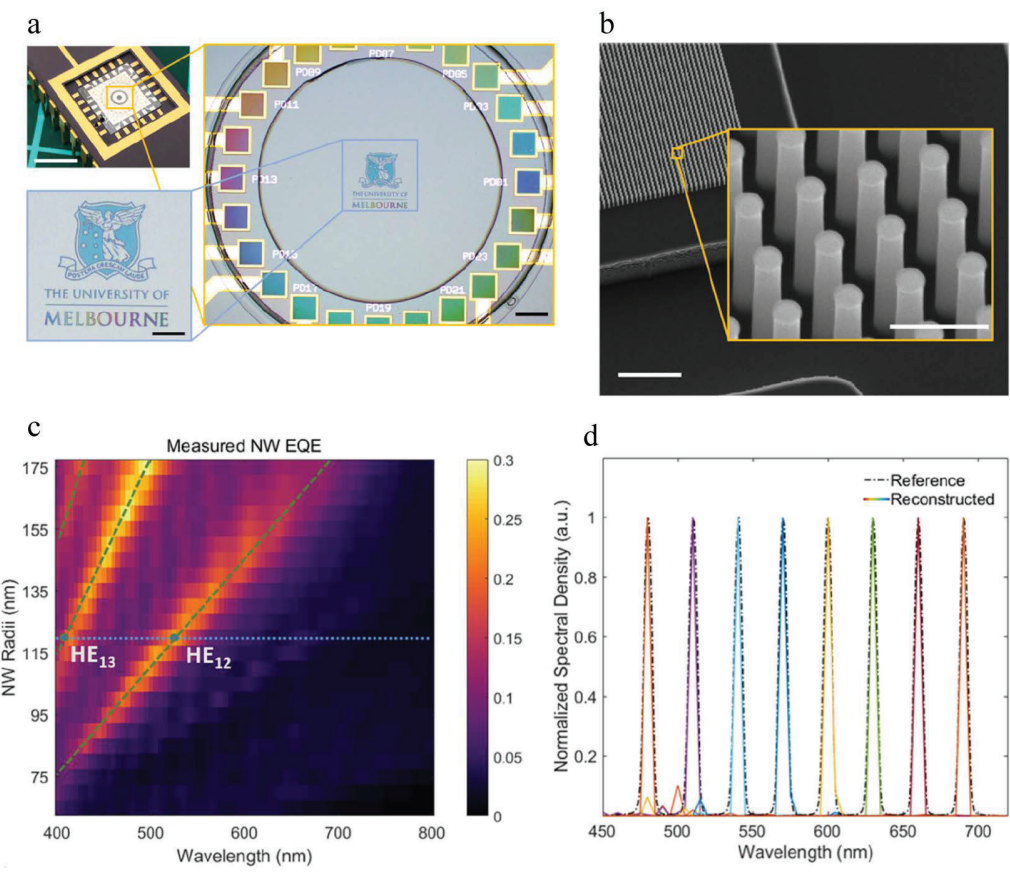

는 


\section{University Library}

\section{- M M N E R VA A gateway to Melbourne's research publications}

Minerva Access is the Institutional Repository of The University of Melbourne

Author/s:

Ye, M;Zha, J;Tan, C;Crozier, KB

Title:

Graphene-based mid-infrared photodetectors using metamaterials and related concepts

Date:

2021-09-01

Citation:

Ye, M., Zha, J., Tan, C. \& Crozier, K. B. (2021). Graphene-based mid-infrared photodetectors using metamaterials and related concepts. APPLIED PHYSICS REVIEWS, 8 (3), https:// doi.org/10.1063/5.0049633.

Persistent Link:

http://hdl.handle.net/11343/294843 